OPEN ACCESS

Edited by:

Xanthe Vafopoulou, York University, Canada

Reviewed by:

Claire Donald

University of Glasgow,

United Kingdom

Jeffrey Wilusz,

Colorado State University,

United States

Sonja Hall-Mendelin,

Queensland Health, Australia

*Correspondence:

Jisheng Liu

jisheng.liu@gzhu.edu.cn

Luc Swevers

swevers@bio.demokritos.gr

${ }^{\dagger}$ These authors have contributed equally to this work

Specialty section: This article was submitted to Invertebrate Physiology, a section of the journa

Frontiers in Physiology

Received: 02 April 2019 Accepted: 27 June 2019

Published: 11 July 2019

Citation:

Liu J, Swevers L, Kolliopoulou A and Smagghe G (2019) Arboviruses

and the Challenge to Establish

Systemic and Persistent Infections

in Competent Mosquito Vectors:

The Interaction With the RNA

Mechanism. Front. Physiol. 10:890.

doi: 10.3389/fphys.2019.00890

\section{Arboviruses and the Challenge to Establish Systemic and Persistent Infections in Competent Mosquito Vectors: The Interaction With the RNAi Mechanism}

\author{
Jisheng Liu' ${ }^{1 * \dagger}$, Luc Swevers ${ }^{2 * \dagger}$, Anna Kolliopoulou² and Guy Smagghe ${ }^{3}$ \\ 'School of Life Sciences, Guangzhou University, Guangzhou, China, ${ }^{2}$ Institute of Biosciences and Applications, National \\ Centre of Scientific Research "Demokritos", Athens, Greece, ${ }^{3}$ Department of Plants and Crops, Faculty of Bioscience \\ Engineering, Ghent University, Ghent, Belgium
}

Arboviruses are capable to establish long-term persistent infections in mosquitoes that do not affect significantly the physiology of the insect vectors. Arbovirus infections are controlled by the RNAi machinery via the production of viral siRNAs and the formation of RISC complexes targeting viral genomes and mRNAs. Engineered arboviruses that contain cellular gene sequences can therefore be transformed to "viral silencing vectors" for studies of gene function in reverse genetics approaches. More specifically, "ideal" viral silencing vectors must be competent to induce robust RNAi effects while other interactions with the host immune system should be kept at a minimum to reduce non-specific effects. Because of their inconspicuous nature, arboviruses may approach the "ideal" viral silencing vectors in insects and it is therefore worthwhile to study the mechanisms by which the interactions with the RNAi machinery occur. In this review, an analysis is presented of the antiviral RNAi response in mosquito vectors with respect to the major types of arboviruses (alphaviruses, flaviviruses, bunyaviruses, and others). With respect to antiviral defense, the exo-RNAi pathway constitutes the major mechanism while the contribution of both miRNAs and viral piRNAs remains a contentious issue. However, additional mechanisms exist in mosquitoes that are capable to enhance or restrict the efficiency of viral silencing vectors such as the amplification of RNAi effects by DNA forms, the existence of incorporated viral elements in the genome and the induction of a non-specific systemic response by Dicer-2. Of significance is the observation that no major "viral suppressors of RNAi" (VSRs) seem to be encoded by arboviral genomes, indicating that relatively tight control of the activity of the RNA-dependent RNA polymerase (RdRp) may be sufficient to maintain the persistent character of arbovirus infections. Major strategies for improvement of viral silencing vectors therefore are proposed to involve engineering of VSRs and modifying of the properties of the RdRp. Because of safety issues (pathogen status), however, 
arbovirus-based silencing vectors are not well suited for practical applications, such as RNAi-based mosquito control. In that case, related mosquito-specific viruses that also establish persistent infections and may cause similar RNAi responses may represent a valuable alternative solution.

Keywords: arbovirus, mosquito, persistent infection, RNAi, siRNA, piRNA, antiviral defense, viral suppressor of RNAi

\section{INTRODUCTION}

RNA interference (RNAi) has become an important tool to analyze gene function in eukaryotes, including insects. RNAi technology is based on the administration of dsRNA that will trigger the degradation of homologous cellular mRNAs. Because of the specificity of gene silencing effects, RNAi has become a powerful reverse genetics tool for analysis of gene function, especially in non-model organisms, which include many insects (Bellés, 2010; Huvenne and Smagghe, 2010; Santos et al., 2014; Schmitt-Engel et al., 2015; Whitten and Dyson, 2017; Lopez et al., 2019). However, in many insects the process of gene silencing following injection or feeding of dsRNA is not very robust which has stimulated research to develop new methods of delivery of dsRNA to increase efficiency (Zhang et al., 2013; Joga et al., 2016). One delivery system that is proposed is based on the use of recombinant viruses that naturally trigger the RNAi response in insects (Kolliopoulou et al., 2017). This strategy, termed virus-induced gene silencing (VIGS), was first pioneered in plants and employs the efficiency by which viruses can enter and replicate in cells. However, a disadvantage of the use of viruses is that robust replication can cause cellular damage and induce the immune response that will obscure the interpretation of the phenotypes that correspond to the host sequences that are integrated in the recombinant viral silencing vectors. It is therefore proposed that viral silencing vectors should be engineered carefully such that a moderate level of replication is achieved, capable to deliver sufficient dsRNA molecules to activate the RNAi machinery, while simultaneously avoiding to interfere with cellular function or to induce the immune response. For this reason, the development of this technique can benefit and learn from the study of arbovirus infections in insect vectors because they have been reported to both interact with the RNAi machinery and to be entirely non-pathogenic (Myles et al., 2008; Blair, 2011; Rückert et al., 2014; Goic et al., 2016). In insect vectors, arboviruses are presumed to strike a delicate balance between replication kinetics and avoidance of the immune response (O'Neal et al., 2014; Samuel et al., 2018). As a complicating factor, avoidance of the immune response regularly includes suppression of RNAi, which makes the engineering of viruses as both efficient and specific silencing vectors particularly challenging.

Arbovirus infections of mosquito vectors represent an interesting system of how persistent and systemic virus infections become established and maintained in insect hosts. As such, studies of arbovirus infections can provide important insights to inspire the optimization of viral silencing vectors in other insects. As a background, this review will investigate the process of RNAi as an antiviral response during arbovirus infections and to what extent arboviruses (in their persistent state) can function as RNAi transduction vectors in mosquitoes. While RNAi is considered the most important factor that modulates arbovirus-mosquito interactions, it should be noted that other pathways and processes (e.g., innate immune response pathways, stress response, apoptosis and autophagy, alternative RNA degradation pathways, microbiome; Kingsolver et al., 2013; Swevers et al., 2018) may also have a significant impact. In this review, focus will be on the RNAi response in which the small interfering RNA (siRNA) pathway ("exo-RNAi"; see section "The RNAi Machinery in Mosquito Vectors") is considered the major defense mechanism together with possible minor contributions from the PIWI-associated RNA (piRNA) and microRNA (miRNA) mechanisms.

\section{THE NATURE OF ARBOVIRUS INFECTIONS IN MOSQUITO VECTORS}

Arboviruses or arthropod-borne viruses refer to a non-taxonomic group of viruses that are transmitted to humans and livestock by arthropod vectors, typically insects (mosquitoes, sandflies, black flies, biting midges; all Diptera) and ticks (Acari, Arachnida). In this review, most attention is focused on mosquitoes because of the wealth of information that is available, while other insect or tick vectors are occasionally also mentioned if relevant information is available for discussion.

Most arboviruses are RNA viruses and characterized by monopartite linear $(+)$ ssRNA genome [Togaviridae (genus Alphavirus), Flaviviridae], monopartite linear (-) ssRNA genome (Rhabdoviridae), segmented linear $(-)$ or ambisense ssRNA genome (Bunyavirales) and segmented linear dsRNA genome (Reoviridae). While arboviruses can cause some of the most devastating diseases in humans (hemorrhagic disease and encephalitis-like illnesses), a distinguishing feature of arbovirus infection in mosquitoes is the establishment of a non-pathogenic, persistent state (Lambrechts and Scott, 2009). During the acute phase of infection, viral replication initially can reach high levels but viral titers subsequently become modulated to low levels during the phase of persistence (Myles et al., 2008; Fragkoudis et al., 2008). For establishment of the persistent state, a delicate balance between the virus and the immune system of the mosquito is necessary to regulate replication and maintain viral presence without causing significant adverse effects that could affect the transmission efficiency to a new host. To optimize transmission, it is indeed essential that arbovirus infections do 
not result in a decrease of mosquito survival because mortality before completing the transmission cycle is predicted to have a large impact on vectorial capacity (Black and Moore, 2005).

Mosquito vector competence is defined as the overall capacity of the insect vector to become orally infected by the virus after a blood meal and to transmit the virus to the next vertebrate host (Smith et al., 2012). Arboviruses therefore must be capable to infect and replicate in the midgut epithelial cells of the mosquito vector, to escape from the midgut cells to disseminate in the hemolymph and secondary insect tissues (where secondary viral replication occurs), and finally to infect the salivary glands from which progeny virus in the saliva is transmitted to the vertebrate host during a blood meal (Hardy et al., 1983; Franz et al., 2015). Depending on the particular virus-mosquito vector pair, dissemination of virus from the midgut to other tissues typically is observed between 3 and 7 days post-infection (p.i.), while mosquitoes become competent for virus transmission through the saliva between 5 and 14 days p.i. (Ebel et al., 2005; Carissimo et al., 2015; Rückert et al., 2017). Virus can persist for periods up to 4 weeks in midgut and salivary glands of infected mosquitoes (Girard et al., 2005). The "extrinsic incubation period", defined as the interval between acquisition and transmission, is thought to be modulated extensively by the antiviral immune response, which includes RNAi, in the mosquito vector (Cheng et al., 2016).

\section{THE RNAi MACHINERY IN MOSQUITO VECTORS}

Insects are characterized by three RNAi pathways (Campbell et al., 2008; Blair, 2011; Donald et al., 2012; Rückert et al., 2014; O'Neal et al., 2014; Dowling et al., 2016). An overview of the three RNAi pathways that is focused on aedine mosquitoes is presented in Figure 1 and discussed below.

MicroRNAs (miRNAs) originate from nuclear genes and regulate cellular gene expression at the posttranscriptional level. The miRNA machinery is conserved among insects and consists of Drosha and Pasha, that process the primary miRNA transcripts in the nucleus; Dicer-1 (Dcr-1) and Loquacious (Loqs), that carry out further processing to generate $\sim 22 \mathrm{nt}$ mature miRNAs in the cytoplasm; and Argonaute-1 (Ago-1) that constitutes the central factor in the miRNA-induced silencing complex (miRISC).

The main function of the siRNA pathway is the defense against invading nucleic acids such as viruses (called "exo-RNAi" because of the exogenous origin of the dsRNA trigger) and transposable elements in the genome ("endo-RNAi"). In this pathway, long dsRNAs are processed by Dicer-2 (Dcr-2) and its co-factor R2D2 (or a specific isoform of Loqs in the case of the defense against transposons) to $\sim 21 \mathrm{nt}$ siRNAs which are subsequently loaded into siRISC containing Argonaute-2 (Ago-2) to silence viral genes and transposons. Factors in the siRNA pathway have undergone accelerated evolution as a consequence of constant adaptations during the host-virus arms race (Obbard et al., 2006). Mosquito species that are commonly used in research, such as Aedes aegypti (yellow fever mosquito), Aedes albopictus (Asian tiger mosquito) and Anopheles gambiae (African malaria mosquito), are characterized by single genes each of $d c r-2$ and ago-2, while a duplication of ago-2 has occurred in the culicine mosquito Culex pipiens (Campbell et al., 2008; Lewis et al., 2016).

The third pathway, PIWI-interacting RNA (piRNA) pathway, was initially identified as a defense mechanism against transposition of mobile elements in the germline of Drosophila (Handler et al., 2013). Biogenesis of 24-29 nt piRNAs occurs in a Dicer-independent manner and the effector RISC complexes in the piRNA pathway employ members of the PIWI subclass of Argonaute proteins [Piwi, Aubergine (Aub) and Ago-3 in Drosophila] (Miesen et al., 2016b). The piRNA pathway in Drosophila consists of two branches: (1) the primary (intermediate) pathway in which (single-stranded) transcripts from discrete genomic loci termed piRNA clusters (consisting of remnants of transposable elements) are processed by the nuclease Zucchini, followed by loading of piRNAs in Piwi-containing effector complexes to perform transcriptional gene silencing in the nucleus; and (2) the secondary (mature) pathway that occurs in the cytoplasm and is characterized by the ping-pong amplification loop. In the ping-pong mechanism, transposon (sense) transcripts are cleaved by Aub (loaded with antisense piRNAs) to generate complementary piRNAs (sense orientation) that are loaded in Ago-3-complexes. Because sense piRNA-Ago-3 complexes will subsequently target again transposon sequences in the antisense orientation, an amplification loop is created of production of both mature antisense and sense piRNAs that expedite transposon silencing. In the ping-pong mechanism, the $5^{\prime}$-end of the guide piRNA is located at exactly $10 \mathrm{nt}$ from the $5^{\prime}$-end of the cleavage site. Because the $5^{\prime}$-ends of piRNAs associated with Piwi or Aub have a characteristic U-bias, products are generated with $\mathrm{A}$ at the 10th position, that become regularly associated with Ago-3. The U1/A10 bias and the $10 \mathrm{nt}$ overlap between the $5^{\prime}$-ends in complementary small RNAs are therefore regarded as hallmarks of the ping-pong mechanism in the piRNA pathway (Senti and Brennecke, 2010).

However, while the piRNA pathway is mainly restricted to the germline in Drosophila, research in insects and other arthropods revealed an ancestral role for piRNAs in defense against transposable elements in both somatic and germline tissues (Lewis et al., 2018). Furthermore, while ago-3 is conserved as a single gene in many insects, the lineage of piwi and $a u b$ is characterized by a high rate of duplication events in dipteran insects that reflects the evolutionary arms race against transposable elements (Lewis et al., 2016). More specifically, the existence of piwi and $a u b$ genes (that have separate functions in transcriptional and post-transcriptional silencing, respectively) in Drosophila reflects the duplication of an ancestral gene ("piwi/aub") at the base of the Brachycera. In aedine and culicine mosquitoes, on the other hand, the hypothetical "piwi/aub" ancestral gene has undergone a remarkable expansion (seven genes in Ae. aegypti, nine genes in Ae. albopictus, and six genes in Cx. pipiens quinquefasciatus) (Campbell et al., 2008; Lewis et al., 2016; Wang et al., 2018). By contrast, the genome of $A n$. gambiae encodes one ago-3 and two Piwi-related genes, named ago-4 and ago-5, that are orthologs of piwi/aub (Campbell et al., 2008; Vodovar et al., 2012; Macias et al., 2014). Also the genome of the midge, Culicoides sonorensis (Ceratopogonidae) encodes one ago-3 and two "aub/piwi" genes (Lewis et al., 2016). 


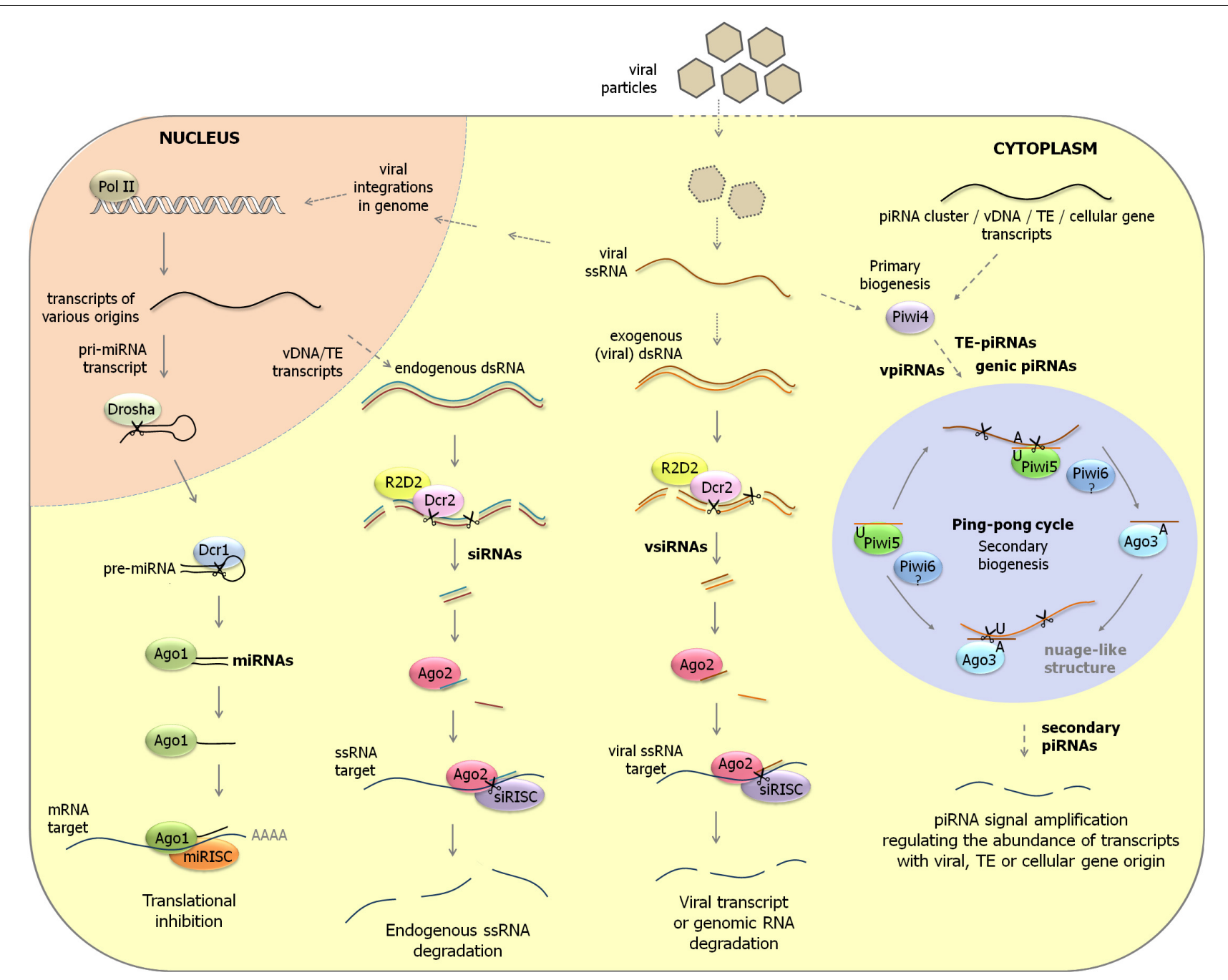

FIGURE 1 | Overview of RNAi pathways in aedine mosquitoes. (Left) miRNA pathway. Primary miRNA transcripts are processed by Drosha in the nucleus to pre-miRNAs. After their transport to the cytoplasm, pre-miRNAs undergo further cleavage by Dicer-1 to mature miRNAs. miRISC complexes containing Ago-1 regulate cellular gene expression by translation inhibition after hybridization to mRNA targets. (Middle) siRNA pathway. Long dsRNA precursors that have endogenous (transposable elements, viral DNA forms) or exogenous (viral replication intermediates) origin are cleaved by Dicer-2 and its co-factor R2D2 to siRNAs and vsiRNAs. siRISC complexes containing Ago-2 subsequently scan parasitic RNA populations (transposon transcripts, viral transcripts and genomic RNAs, RNAs derived from viral integrations in genome) to trigger their destruction. (Right) piRNA pathway. In aedine mosquitoes, an expansion of PIWI-class Argonaute genes is observed which are expressed in somatic tissues and are involved in transposon control but possibly also in antiviral defense and cellular gene regulation. ssRNA precursors from various origins (transposable elements, viral mRNAs and genomic RNAs, transcripts from viral DNA forms, cellular gene transcripts) are processed to primary piRNAs by a Dicer-independent mechanism. While Piwi-4 does not directly interact with piRNAs, it was proposed that it acts as an important factor to activate the production of secondary piRNAs by the ping-pong mechanism. In the ping-pong cycle, piRNAs of antisense orientation (U1 bias) are mostly associated with Piwi-5 and possibly also with Piwi-6. On the other hand, piRNAs of sense orientation (A10 logo) are loaded by Ago-3. The piRNA ping-pong cycle is considered an important amplification mechanism to regulate the abundance of transcripts of transposon, viral or cellular origin. pri-miRNA, primary miRNA; pre-miRNA, precursor miRNA; vDNA, viral DNA form; TE, transposable element.

More specific data with respect to expression of PIWI subclass genes in tissues of mosquitoes are available for Ae. aegypti and Ae. albopictus. Of the seven Piwi-related genes and one ago-3 gene that are identified in the genome of Ae. aegypti, piwi-4, piwi-5, piwi-6, and ago-3 are clearly expressed in the somatic tissues of the mosquito (Morazzani et al., 2012; Miesen et al., 2015). On the other hand, piwi-1-3 expression seems to be germline specific while piwi-7 is only present in the early embryo (Akbari et al., 2013). A recent study shows increased expression of Piwi-related and ago-3 genes in ovarian tissue compared to midgut in $C x$. quinquefasciatus mosquitoes (Rückert et al., 2019).

The expansion of PIWI subclass genes in the genome of Ae. albopictus consists of two ago-3 homologs, six homologs of ago4 of An. gambiae (piwi-1-6, related to Ae. aegypti piwi-1-4) and three homologs of ago-5 of An. gambiae (piwi-7-9, related to Ae. aegypti piwi-5-7) (Wang et al., 2018). In Ae. albopictus adult females, mRNAs of the two ago-3 paralogs and piwi-1-7 can be readily detected while piwi- 8 and -9 are highly expressed in 
embryos. In midgut tissue, only mRNAs of piwi-5-7 and the two ago-3 paralogs are present.

\section{EVIDENCE OF INTERACTIONS OF ARBOVIRUSES WITH THE RNAi MACHINERY}

Even before the process of RNAi was clarified in animals (Fire et al., 1998), it was observed that double subgenomic recombinant Sindbis viruses (SINV; Alphavirus) that express sequences of genetically unrelated RNA viruses could induce resistance against infection of these viruses in mosquito cells (Olson et al., 2002). This strategy, called "pathogen-derived resistance" or "RNA-mediated cross-protection between viruses," could provide protection against infection of dengue virus (DENV; Flaviviridae) (Gaines et al., 1996; Olson et al., 1996; Adelman et al., 2001) as well as La Crosse virus (LACV; Bunyavirales) (Powers et al., 1996). Pathogen-derived resistance was sequence-specific, since it was effective against one specific DENV serotype while other serotypes were not affected, and it was mediated at the level of RNA since the use of antisense constructs or the introduction of artificial stop codons in sense constructs did not affect efficiency. The process is now understood to be triggered by RNAi in which viral replication intermediates with dsRNA structure are recognized by Dicer to generate siRNAs that can target the genetically unrelated viruses (in addition to targeting the SINV viruses) (Bronkhorst and van Rij, 2014; Gammon and Mello, 2015). This phenomenon was observed in both mosquitoes (Ae. aegypti and Ae. triseriatus) as well as in C6/36 cells that are derived from Ae. albopictus (Gaines et al., 1996; Powers et al., 1996; Olson et al., 2002).

The sensitivity of arbovirus infections to silencing by the RNAi mechanism was also demonstrated in transformed C6/36 cell lines that express an inverted repeat RNA corresponding to the prM gene of DENV-2 (Adelman et al., 2002). Eight out of 18 transformed C6/36 cell lines that expressed the inverted repeat RNA were resistant to DENV-2 challenge which was correlated with the production of small RNAs from the hairpin construct. This strategy was subsequently further extended to generate transgenic Ae. aegypti mosquitoes that expressed an RNA hairpin targeting the prM region of DENV-2 and that were shown to be refractory to DENV-2 infection (Franz et al., 2006, 2014). Induction of RNA hairpin expression by the midgut-specific carboxypeptidase A promoter occurred after feeding of a blood meal and was shown to provide protection against DENV-2 infection by oral feeding. The involvement of the RNAi mechanism was confirmed following the detection of mainly $21 \mathrm{nt}$ siRNAs originating from the hairpin and by the loss of protection against DENV-2 infection following knock-down of ago-2. Protection was strong against DENV2 infection but not against other DENV serotypes or CHIKV (Alphavirus), confirming the specificity of the antiviral RNAi response. Introgression of the RNA hairpin transgene into mosquitoes of another genetic background resulted in a change from the susceptible to the refractory phenotype (Franz et al., 2014). A homozygous Ae. aegypti mosquito line that is refractory to DENV-2 infection with minimal fitness loss was selected that can be used for studies of spread within mosquito populations. Furthermore, a complementary study that used transgenic mosquitoes that express an RNA hairpin targeting prM of DENV2 in the female salivary glands likewise resulted in inhibition of viral infection of the salivary glands as well as in a significant diminution of the transmission of DENV-2 (Mathur et al., 2010).

\section{ANTIVIRAL DEFENSE BY THE EXO-RNAi PATHWAY IN MOSQUITOES}

Interactions of viral infections with the RNAi machinery in Drosophila are usually assessed by three criteria: (1) the production of viral siRNAs (vsiRNAs); (2) increase in viral replication and mortality in ago-2 and dcr-2 mutants; (3) the presence of genes in the viral genome that encode VSRs (Bronkhorst and van Rij, 2014; Pijlman, 2014). When these criteria are applied to mosquitoes, two difficulties are encountered: (1) a $d c r-2$ mutant mosquito line only recently has become available for Ae. aegypti (Samuel et al., 2016; Varjak et al., 2017b) and therefore the assessment of the involvement of ago-2 and $d c r-2$ in earlier studies and in other mosquitoes was carried out by RNAi-mediated knock-down, which is considered an inefficient process; and (2) arboviruses in general are considered to be lacking VSR genes; although a few convincing candidates were proposed, conclusive evidence within the native viral context or in the natural vector was not achieved (O’Neal et al., 2014; Samuel et al., 2018). Although the evidence is less robust as in Drosophila, the involvement of the exo-RNAi pathway in the defense against arboviral infections in mosquitoes nevertheless does not seem to be in doubt. However, research has also indicated that the efficiency of RNAmediated silencing can be modulated by environmental factors, such as temperature, with repercussions on the antiviral response, e.g., the higher arbovirus infection levels observed at cooler temperature (Adelman et al., 2013).

When viral small RNAs (vsiRNAs but also viral piRNAs; see section "What is the Role of the piRNA Pathway in Antiviral Defense?"), obtained after deep sequencing analysis, are mapped to the viral genomes, cold and hot spot regions of under- and over-representation, respectively, are typically observed. The occurrence of these regions may result from preferential processing by Dcr-2 but other causes, such as differential stability of small RNAs and preferential targeting of regions for production of viral DNA forms (considered as an amplification mechanism for viral small RNA production; see section "Amplification of the Antiviral RNAi Response and Potential Establishment of Immune Memory") need to be considered. Moreover, the preferential detection may be the result of technical issues that occur in deep sequencing experiments, such as library preparation (Linsen et al., 2009). Ideally, the abundance of viral small RNAs corresponding to specific regions of the viral genome should be confirmed by other biochemical or molecular methods (e.g., Northern blot, qPCR).

Below follows a detailed analysis of the function of the exoRNAi pathway as an antiviral defense mechanism in mosquitoes. 
Production of vsiRNAs is summarized in Table 1. An overview of the documented involvement of factors in the exo-RNAi pathway is presented in Table 2 .

\section{Alphaviruses}

The most studied arboviruses of the Alphavirus genus (family: Togaviridae) are Sindbis virus (SINV), Semliki Forest virus (SFV), Chikungunya virus (CHIKV) and o'nyong-nyong virus (ONNV). Alphaviruses are characterized by a single linear $(+)$ ssRNA genome $(11-12 \mathrm{~kb})$ that also serves as the mRNA for the non-structural polyprotein while a subgenomic RNA produced during infection functions as the source for the structural proteins. Under natural conditions, SINV circulates between Culex mosquitoes and birds, while humans act as "dead-end" hosts. Both SFV and CHIKV are transmitted to humans by Aedes species while the primary vectors of ONNV are anopheline mosquitoes (Atkins, 2013).

\section{SINV}

Although SINV is not naturally transmitted by A. aegypti, particular genotypes can establish persistent midgut infections after an infectious blood meal and spread to other tissues at high frequency (Campbell et al., 2008). RNAi-mediated knockdown of ago-2, dcr-2 and to a lesser extent, the RISC component Tudor staphylococcal nuclease (TSN), resulted in enhancement of viral infection by feeding but did not affect mortality. Increased replication of recombinant SINV vectors was also observed in dcr-2 null mutant Ae. aegypti mosquitoes (and was associated with increased mortality; Samuel et al., 2016). Injection of dsRNA targeting the viral non-structural protein nsP3, on the other hand, provided strong protection against viral infection. Interestingly, different strains of recombinant SINV exhibited differences in the rate of viral replication which could be negatively correlated with the production of viral small RNAs (Campbell et al., 2008). Components of the RNAi machinery (Ago-2, Dcr-2, TSN) showed a transcriptional response after ingestion of a blood meal

TABLE 1 | Viral small RNAs produced during arbovirus infections of mosquitoes.

\begin{tabular}{|c|c|c|c|c|}
\hline Virus & Mosquito & Tissue & Viral small RNAs & References \\
\hline \multicolumn{5}{|c|}{ Alphavirus } \\
\hline \multirow[t]{2}{*}{$\mathrm{CHIKV}$} & Ae. aegypti & Whole body & $21 \mathrm{nt} v \mathrm{siRNA}$ & Morazzani et al., 2012 \\
\hline & & & 26-29 nt vpiRNA & \\
\hline \multirow[t]{2}{*}{ CHIKV } & Ae. albopictus & Whole body & $21 \mathrm{nt}$ vsiRNA & Morazzani et al., 2012 \\
\hline & & Head-thorax & 26-29 nt vpiRNA & \\
\hline \multirow[t]{2}{*}{$\mathrm{CHIKV}$} & Ae. albopictus & Whole body & $21 \mathrm{nt} v \mathrm{siRNA}$ & Goic et al., 2016 \\
\hline & & & 27-29 nt vpiRNA & \\
\hline ONNV & An. gambiae & Whole body & $21 \mathrm{nt}$ vsiRNA & Myles et al., 2009 \\
\hline ONNV & An. gambiae & Whole body & $21 \mathrm{nt}$ vsiRNA & Carissimo et al., 2015 \\
\hline SINV & Ae. aegypti & Whole body & $21 \mathrm{nt}$ vsiRNA & Myles et al., 2008 \\
\hline SINV & Cx. pipiens & Whole body & $21 \mathrm{nt}$ vsiRNA & Miesen et al., 2016b \\
\hline \multicolumn{5}{|c|}{ Flavivirus } \\
\hline DENV & Ae. aegypti & Whole body & $21 \mathrm{nt}$ vsiRNA & Scott et al., 2010 \\
\hline \multirow[t]{3}{*}{ DENV } & Ae. aegypti & Whole body & $<20$ nt usRNA & Hess et al., 2011 \\
\hline & & & 20-23 nt vsiRNA & \\
\hline & & & 24-30 nt vpiRNA & \\
\hline \multirow[t]{3}{*}{ DENV } & Ae. albopictus & Whole body & 13-19 nt usRNA & Wang et al., 2018 \\
\hline & & Midgut & 20-23 nt vsiRNA & \\
\hline & & & 24-30 nt vpiRNA & \\
\hline USUV & Cx. pipiens & Whole body & 21 nt vsiRNA & Fros et al., 2015 \\
\hline WNV & Ae. aegypti & Midgut & 21 nt vsiRNA & Rückert et al., 2019 \\
\hline WNV & Cx. pipiens quinquefaciatus & Midgut & 20-22 nt vsiRNA & Brackney et al., 2009 \\
\hline WNV & Cx. pipiens & Whole body & 21 nt vsiRNA & Fros et al., 2015 \\
\hline WNV & Cx. pipiens & Whole body & $21 \mathrm{nt}$ vsiRNA & Göertz et al., 2016 \\
\hline WNV & Cx. quinquefasciatus & $\begin{array}{l}\text { Midgut, } \\
\text { salivary glands }\end{array}$ & 21 nt vsiRNA & Rückert et al., 2019 \\
\hline \multicolumn{5}{|c|}{ Bunyaviridae } \\
\hline \multirow[t]{2}{*}{ RVFV } & Ae. aegypti & Whole body & $21 \mathrm{nt}$ vsiRNA & Dietrich et al., 2017a \\
\hline & & & 25-30 vpiRNA & \\
\hline \multirow[t]{2}{*}{ RVFV } & Ae. vexans & Whole body & $21 \mathrm{nt}$ vsiRNA & Dietrich et al., 2017a \\
\hline & & & 27-30 vpiRNA & \\
\hline \multirow[t]{3}{*}{ RVFV } & Cx. quinquefasciatus & Whole body & 19 nt usRNA & Dietrich et al., 2017a \\
\hline & & & $21 \mathrm{nt}$ vsiRNA & \\
\hline & & & 25-29 vpiRNA & \\
\hline
\end{tabular}


TABLE 2 | RNAi factors that affect arbovirus replication in mosquitoes.

\begin{tabular}{|c|c|c|c|c|}
\hline Virus & Mosquito & RNAi factor & Phenotype & References \\
\hline \multicolumn{5}{|c|}{ Alphavirus } \\
\hline $\mathrm{CHIKV}$ & Ae. aegypti & Ago-2 (RNAi knockdown) & Increase in viral titer (midgut and head) & McFarlane et al., 2014 \\
\hline ONNV & An. gambiae & $\begin{array}{l}\text { Ago-2, Ago-3 (no effect for Ago-1, } \\
\text { Ago-4, Ago-5) (RNAi knockdown) }\end{array}$ & Increase in viral titers & Keene et al., 2004 \\
\hline ONNV & An. gambiae & Ago-2 (RNAi knockdown) & Increase in viral titer (infection by injection) & Waldock et al., 2012 \\
\hline ONNV & An. gambiae & Ago-2 (RNAi knockdown) & Increase in systemic infection (infection by feeding) & Carissimo et al., 2015 \\
\hline SINV & Ae. aegypti & $\begin{array}{l}\text { Ago-2, Dcr-2, TSN (RNAi } \\
\text { knockdown) }\end{array}$ & Increased viral replication (mortality not affected) & Campbell et al., 2008 \\
\hline SINV & Ae. aegypti & $d c r-2$ mutant & $\begin{array}{l}\text { Increased viral replication } \\
\text { Increased mortality }\end{array}$ & Samuel et al., 2016 \\
\hline SINV & Ae. aegypti & $\begin{array}{l}\text { Dcr-2 (induction of RNA hairpin in } \\
\text { midgut upon feeding) }\end{array}$ & $\begin{array}{l}\text { Increased viral titers } \\
\text { Increased infection of midgut } \\
\text { Increased dissemination of virus to other tissues }\end{array}$ & Khoo et al., 2010 \\
\hline \multicolumn{5}{|c|}{ Flavivirus } \\
\hline DENV & Ae. aegypti & $\begin{array}{l}\text { Dcr-2, R2D2, Ago-2 (RNAi } \\
\text { knockdown) }\end{array}$ & $\begin{array}{l}\text { Increase in viral titer } \\
\text { Increase in viral transmission }\end{array}$ & Sánchez-Vargas et al., 2009 \\
\hline YFV & Ae. aegypti & $d c r-2$ mutant & Increase in viral replication & Samuel et al., 2016 \\
\hline
\end{tabular}

and after oral viral infection, while also changes in Ago-2 protein levels were observed. Another study observed down-regulation of $d c r-2$ in SINV-infected Ae. aegypti mosquitoes during late infection (Sanders et al., 2005).

Injection of SINV in Ae. aegypti mosquitoes resulted in the production of vsiRNAs predominantly of $21 \mathrm{nt}$ size (Myles et al., 2008). VsiRNAs could be mapped along the length of the viral genome but showed regions of preferential accumulation (hot spots, which are typically observed in all virus infections). A significant bias for the positive strand was also observed, indicating that vsiRNAs derive from both replication intermediates and structured regions in viral RNA transcripts (Campbell et al., 2008; Myles et al., 2008, 2009).

Studies of oral infections of SINV in Ae. aegypti mosquitoes established that the RNAi response in midgut tissue was important to prevent dissemination of the virus to other tissues of the body (Khoo et al., 2010, 2013). Silencing of expression of $d c r-2$ specifically in midgut tissue of Ae. aegypti mosquitoes immediately following an infectious blood meal of recombinant SINV indeed resulted in increased viral titers and increased infection rate of the midgut as well as increased dissemination to other tissues (Khoo et al., 2010). Similar results were obtained in transgenic Ae. aegypti that constitutively express the B2 RNAi inhibitor of Flock house virus (FHV; Nodaviridae; see also section "Evidence for Presence of VSR Genes in Arboviral Genomes" for a discussion of RNAi inhibitors) although in this case only increased dissemination of SINV from midgut to other tissues was observed (Khoo et al., 2013).

\section{ONNV}

Anopheline mosquitoes are mainly known for the transmission of malaria parasites while their vectoring of arboviruses seems limited. One exception is ONNV that is transmitted by $A n$. funestus and An. gambiae mosquitoes (Rezza et al., 2017). When recombinant ONNV with a GFP reporter cassette was injected in An. gambiae mosquitoes, its replication and dissemination could be inhibited by dsRNA targeting the non-structural protein
nsP3 (Keene et al., 2004). On the other hand, silencing of ago-2 (siRNA pathway) and ago-3 (piRNA pathway; see also section "Analysis of the Role of the piRNA Pathway in Antiviral Defense in Mosquito Cell Lines") but not ago-1 (miRNA pathway) or the Piwi/Aub-related ago-4 and ago-5 (piRNA pathway) (Lewis et al., 2016) resulted in an increase in viral titers (Keene et al., 2004).

Injection of ONNV in A. gambiae mosquitoes resulted in the production of $21 \mathrm{nt}$ vsiRNAs that show similar properties to that observed following injection of SINV in Ae. aegypti mosquitoes (discussed above; Campbell et al., 2008; Myles et al., 2008, 2009).

However, in contrast to what is observed during midgut infection of Ae. aegypti mosquitoes with SINV, a more recent study that investigated in more detail the early stages of infection of An. gambiae with ONNV revealed that the RNAi response was not important to control the primary infection of midgut cells with an infectious blood meal [in contrast to the immune deficiency (Imd) and Janus kinase/signal transducer and activator of transcription (JAK/STAT) pathways; Carissimo et al., 2015]. The data also point to complementary immune responses in midgut and systemic compartments since the RNAi response was antiviral during infections after injection of ONNV, in contrast to the JAK-STAT and Imd pathways (Waldock et al., 2012). While vsiRNAs are produced during primary infection of the midgut by ONNV (Carissimo et al., 2015), they do not seem to provide protection at the early stage in the midgut and may function as a signal (rather than an effector) to influence responses in the systemic compartment during later infection.

\section{CHIKV}

When Ae. aegypti and Ae. albopictus mosquitoes were injected with CHIKV, vsiRNAs (21 nt) were produced but also viral small RNAs of piRNA size (23-30 nt) (Morazzani et al., 2012; Goic et al., 2016) (see also section "What is the Role of the piRNA Pathway in Antiviral Defense?" for discussion of the possible role of piRNAs in antiviral defense). However, silencing experiments of genes of the RNAi pathway to determine their effect on CHIKV replication in Ae. aegypti mosquitoes have only been carried out 
for ago-2 for which an antiviral effect was revealed in both midgut and head tissue (McFarlane et al., 2014).

\section{Flaviviruses}

The most studied viruses of the Flavivirus genus (family: Flaviviridae) are DENV (present as four serotypes), West Nile virus (WNV) and Zika virus (ZIKV). Flaviviruses are characterized by a single linear (+) ssRNA genome (10-11 kb) that also functions as mRNA and encodes a single polyprotein that is processed to both structural and non-structural proteins by viral and host proteases. Both DENV and ZIKV (together with the alphavirus CHIKV) are transmitted to humans by Ae. aegypti and Ae. albopictus mosquitoes. The primary vectors for transmission of WNV are Culex mosquitoes with birds as preferential and humans as "dead-end" hosts, respectively (Huang et al., 2014).

\section{DENV}

RNAi modulates DENV replication at different infection stages since knock-down of the siRNA machinery factors $d c r-2, r 2 d 2$ and ago-2 in Ae. aegypti mosquitoes prior to an infectious blood meal affects infection prevalence, dissemination of the virus from the midgut to the salivary glands, viral titer and viral transmission via the saliva (Sánchez-Vargas et al., 2009). Increased expression of $d c r-2$ and ago-2 mRNA was observed during early infection of Ae. aegypti mosquitoes with DENV-2 while during later stages also TSN mRNA was induced (Hess et al., 2011).

Small RNA sequencing revealed vsiRNAs of $21 \mathrm{nt}$ size following oral infection of Ae. aegypti mosquitoes with DENV-2 (Scott et al., 2010; Hess et al., 2011). Nearly equal ratios of positive and negative sense vsiRNA reads were detected suggesting that dsRNA replication intermediates constitute a major source for processing by Dcr-2. However, compared to infections with alphaviruses, the proportion of reads corresponding to vsiRNAs was very low in the case of DENV, possibly indicating low viral replication or effective sequestration of dsRNA triggers in cellular membrane vesicles during replication/transcription (Scott et al., 2010). During oral infection of Ae. albopictus mosquitoes with DENV-2, vsiRNAs (20-23 nt) were detected predominantly in whole body samples, in contrast to other classes of viral small RNAs that were more prevalent in midgut (Wang et al., 2018; see also section "Production of VpiRNAs in Infected Mosquitoes").

\section{WNV}

Midguts of $C x$. pipiens (quinquefasciatus) mosquitoes show a clear RNAi response upon oral infection with WNV (Brackney et al., 2009). The majority of vsiRNAs had a size between 20 and $22 \mathrm{nt}$ with a clear peak of $21 \mathrm{nt}$. RNAi targeting of the positive and negative sense of the WNV genome was proportional to the abundance of the genome strands. However, clear hot spots for generation of vsiRNAs were observed, most notably a 200 nt region in the $5^{\prime}$-part of the Capsid coding sequence, while strongly structured regions in the $3^{\prime}$-UTR were not targeted. Similar profiles of viral small RNAs were also obtained in Culex mosquito bodies after oral infection with WNV that, however, also included targeting of the $3^{\prime}$-UTR region (the site of sfRNA production; see also section "SfRNA"; Fros et al., 2015;
Göertz et al., 2016). A very recent study reported the detection of $21 \mathrm{nt}$ vsiRNAs in midgut and salivary glands following infection of $C x$. quinquefasciatus and Ae. aegypti mosquitoes (Rückert et al., 2019).

\section{ZIKV}

Ae. aegypti mosquitoes that were orally infected with ZIKV showed a clear exo-RNAi response at 7 and 14 days, i.e., the production of $21 \mathrm{nt}$ vsiRNAs that were distributed across the whole genome but showed a slight bias for the positive strand (analysis in whole mosquitoes; Saldaña et al., 2017). Interestingly, knock-down of ago-2 in Ae. aegypti-derived cells did not result in increased viral replication of ZIKV (Varjak et al., 2017b) while only a minor effect was also demonstrated for DENV replication (Miesen et al., 2016a).

\section{Yellow Fever Virus (YFV)}

Replication of YFV was demonstrated to be significantly higher in $d c r-2$ mutant Ae. aegypti (its natural host) compared with wild-type at comparable time points (Samuel et al., 2016). In addition, it was shown that the capsid protein of YFV could act as a VSR in the context of recombinant SINV infections and several biochemical assays (see also section "Evidence for Presence of VSR Genes in Arboviral Genomes” for discussion on VSRs).

\section{Bunyaviruses}

The most studied arboviruses of the Bunyavirales order include Rift Valley Fever virus (RVFV; genus Phlebovirus; Phenuiviridae family), La Crosse virus, Schmallenberg virus and the type species Bunyamwera virus (LACV, SBV and BUNV, respectively; Orthobunyavirus genus; Peribunyavirales family). Bunyaviruses are characterized by a tripartite linear $(-)$ or ambisense ssRNA genome that is organized in three segments, $\mathrm{L}(6-7 \mathrm{~kb}), \mathrm{M}$ $(3.0-4.5 \mathrm{~kb})$, and $\mathrm{S}(1.0-1.7 \mathrm{~kb})$. Several different species of mosquito (e.g., Ae. aegypti, Cx. quinquefaciatus) are able to act as vectors for transmission of RVFV (Linthicum et al., 2016). LACV is maintained in a cycle between its primary vector, Ae. triseriatus, and small mammals, with humans considered as "dead-end" hosts (Beaty et al., 2000). For both RVFV and LACV, vertical transmission to the offspring can occur. The primary vector of BUNV is considered Ae. aegypti, although it can also be transmitted by $A n$. gambiae but not by $C x$. quinquefasciatus (Dutuze et al., 2018). Finally, SBV is an arbovirus believed to be transmitted by midge (Culicoides) species (Schnettler et al., 2013a).

\section{RVFV}

Rift Valley Fever virus infection (through infectious blood meal) of three different mosquito species (Ae. aegypti, Ae. vexans, $C x$. quinquefaciatus) resulted in the production of vsiRNAs that were derived from all three segments with abundance gradient $\mathrm{M}>\mathrm{S}>\mathrm{L}$ and corresponded to genome and antigenome strands in approximately equal ratios (Dietrich et al., 2017a). A hotspot detected at the intergenic region between the $\mathrm{N}$ and Ns genes in the ambisense $S$ segment (observed in mosquito cell lines but to a lesser extent also in Culex mosquitoes) could be caused by hybridization of complementary $\mathrm{N}$ and NSs mRNAs 
(Sabin et al., 2013). However, the abundance of viral small RNAs (including vsiRNAs but also vpiRNAs; see section "What is the Role of the piRNA Pathway in Antiviral Defense?") following infection was observed to be much lower in Culex than in Aedes mosquitoes (Dietrich et al., 2017a). While this could indicate differences in the RNAi response, other explanations are possible, such as infection status, virus-specific effects or co-infection with mosquito-specific viruses (see also section "RNAi and the Antiviral Defense Against Mosquito-Specific Viruses”).

\section{EVIDENCE FOR PRESENCE OF VSR GENES IN ARBOVIRAL GENOMES}

Initially, the prevailing dogma was that arboviruses do not encode VSRs because of the need to establish long-lasting (7-14 days) persistent infections in mosquito vectors such that transmission to the next vertebrate host can be accomplished. The importance to replicate at low levels for maintenance of the persistent state was dramatically demonstrated in experiments using recombinant SINV that expresses the strong RNAi inhibitor protein B2 of FHV (Nodaviridae; Bronkhorst and van Rij, 2014). Injection of SINV-B2 in Ae. aegypti mosquitoes resulted in high mortality concomitantly with high levels of viral replication and a greatly reduced RNAi response (production of $21 \mathrm{nt}$ vsiRNAs; Myles et al., 2008). Similar results were obtained in the Aag-2 cell line (derived from Ae. aegypti) and during oral infections of mosquitoes with SINV-B2 (Cirimotich et al., 2009). Altogether, these results suggest that at least SINV does not encode a VSR because of the need to maintain the persistent character of the infection. The absence of a VSR gene in alphaviruses was confirmed by the observation that infections of SFV did not affect gene silencing of a reporter construct in the U4.4 mosquito cell line (Attarzadeh-Yazdi et al., 2009). Infection of An. gambiae with recombinant ONNV expressing B2 from Nodamura virus (Nodaviridae) by both injection and feeding also resulted in increases in mortality and viral titers (Myles et al., 2008).

Recombinant SINV was subsequently used to test candidate VSRs from other viruses, including other arboviruses (Samuel et al., 2016). Using this system, it was established that the capsid protein of YFV (Flaviviridae) possesses strong VSR activity. VSR activity could also be demonstrated for capsid proteins of other flaviviruses infecting different hosts (ZIKV and DENV-2 naturally infecting Ae. aegypti and humans; WNV infecting Culex mosquitoes and birds; Rio Bravo virus (RBV) isolated from bats but with unknown vector) (Samuel et al., 2016). Furthermore, the decreased replication of wild-type SINV compared to recombinant SINV expressing capsid of YFV can be rescued in Ae. aegypti mosquitoes that are mutant for $d c r$ 2. Biochemical tests demonstrate that the capsid protein of YFV interferes with Dicer processing through binding of long dsRNA (but not siRNA) (Samuel et al., 2016). While no VSR activity could be demonstrated using the recombinant SINV system for other candidates such as subgenomic flaviviral RNA (sfRNA) of WNV, NS4B of DENV or NSs of BUNV, indications of such VSR activity were suggested using other experimental procedures or biochemical assays, as outlined in the following paragraphs.
While alphaviruses (SINV, SFV) are generally not considered to encode VSRs (and actually cause mortality when engineered to express an exogenous RNAi inhibitor; see above), a recent study nevertheless showed that the non-structural proteins nsP2 and nsP3 of CHIKV can inhibit dsRNA- and siRNA-mediated silencing in insect, mammalian and plant cells (Mathur et al., 2016). VSR activity presumably occurs through dsRNA/siRNA binding by RNA-binding motifs present in the helicase domain of nsP2 and the N-terminal macrodomain of nsP3.

\section{SfRNA}

During flaviviral infections, accumulation of sfRNA, an abundant non-coding subgenomic sfRNA representing the last $525 \mathrm{nt}$ of the $3^{\prime} \mathrm{UTR}$, is observed as a result of incomplete degradation of the flaviviral genome by the $5^{\prime}-3^{\prime}$ exoribonuclease XRN1 located in processing bodies (PBs) in mammalian cells, where also the RNAi machinery is located (Pijlman, 2014; Roby et al., 2014). Incomplete degradation is caused by stalling of XRN1 at the $3^{\prime}$ UTR that is characterized by a high degree of secondary structure.

In contrast to the non-structural proteins or the capsid protein of WNV, sfRNA was capable to suppress gene silencing in mammalian cells using reporter assays (Schnettler et al., 2012). Suppression of silencing by sfRNA was also observed in Ae. albopictus U4.4 cells and Drosophila S2 cells. Engineering of the alphavirus SFV with sfRNA of WNV increased its replication in mosquito cells (in apparent contrast to the absence of effects during recombinant SINV infections of Ae. aegypti mosquitoes; see above) while reporter assays established that sfRNA could interfere with both siRNA- and miRNA-mediated silencing in insect cells (Schnettler et al., 2012). In WNV-infected Cx. pipiens mosquito strains, sequencing results show that specific hot spots of vsiRNAs derive from structured regions in the $3^{\prime}$-UTR, indicating that sfRNA might be processed by the RNAi machinery in vivo (Göertz et al., 2016). However, overall vsiRNA levels and profiles are similar in infections of wild-type WNV and WNV deficient for full-length sfRNA (sfRNA1) production, indicating that sfRNA1 does not interfere with the RNAi response, although unique hot spots of vsiRNAs corresponding to sfRNA1 were identified in infections with wild-type WNV (Göertz et al., 2016).

Interestingly, it was demonstrated that production of sfRNA is a key factor to overcome the midgut barrier in Culex mosquitoes during oral infections of WNV (Göertz et al., 2016). On the other hand, transmission and infection rates were not affected for sfRNA-deficient WNV after intrathoracic injection and deficient sfRNA production also did not affect growth rates in mosquito cell lines. It is not clear whether the requirement for sfRNA to overcome the midgut barrier is related to its possible role as a VSR.

Investigations regarding RNAi inhibitory activity of sfRNA were extended to CX. quinquefasciatus mosquitoes infected with either wild-type Kunjin virus (KUNV; Flaviviridae) or a mutant form that is defective for production of sfRNA (Moon et al., 2015). While the amount of viral genomic RNA in infected mosquitoes did not differ between wild-type or mutant KUNV (weak) suppression of dsRNA-mediated gene silencing of the endogenous chymotrypsin gene was only observed during infection with wild-type KUNV, therefore associating production 
of sfRNA with inhibition of RNAi. In the same study (Moon et al., 2015), sfRNA was found to be associated with both Dicer and AGO2 in human cells, therefore implicating a role for sfRNA as an RNA decoy for the (human) dsRNA-binding proteins Dicer and AGO2.

Similar experiments demonstrated also suppressor of RNA silencing activity for DENV-1 sfRNA in Ae. albopictus U4.4 cells (Schnettler et al., 2012. Furthermore, expression of sfRNAs of tick-borne arboviruses was reported to inhibit RNAi in tickderived culture cells (Schnettler et al., 2014).

For its proposed VSR activity, it is hypothesized that sfRNA acts as an RNA decoy and inhibits RNAi by oversaturating Dicer enzyme (Pijlman, 2014; Göertz et al., 2016). In mammalian cells, it was indeed demonstrated that siRNAs can bypass the inhibitory effect of sfRNA, indicating that the function of RISC complexes was not affected (Schnettler et al., 2012).

\section{NS4B}

Also in apparent contrast with the results of the recombinant SINV system (Samuel et al., 2016), another study showed that the NS4B protein of DENV could function as a strong RNAi suppressor, in addition to its role in alfa/beta interferon inhibition (Kakumani et al., 2013). While most experiments were carried out using mammalian cells, it is noted that VSR activity of the non-structural protein NS4B was also demonstrated in Sf21 RNAi sensor cells, a transformed insect (lepidopteran) cell line. NS4B is a small transmembrane protein with demonstrated functions involved in RNA replication and interference with the interferon response while the mechanism by which NS4B could inhibit RNAi remains uncharacterized.

\section{NSs}

In mammalian cells, NSs protein from LACV exhibited VSR activity in reporter assays (Soldan et al., 2005). In addition, BUNV that are deficient for NSs exhibited lower replication than wildtype BUNV in a mosquito cell line that is RNAi competent (U4.4; derived from Ae. albopictus) while no effect was observed in RNAi-deficient cells (C6/36 and C7/10; also derived from Ae. albopictus) (Szemiel et al., 2012) (see also section "Analysis of the Role of the piRNA Pathway in Antiviral Defense in Mosquito Cell Lines" for a discussion of RNAi effects in mosquito cell lines). The exclusive observation of the effect of deletion of NSs on viral replication in the cell line with an intact RNAi mechanism was interpreted as evidence for NSs acting as VSR. However, another study failed to show RNAi suppressor activity for NSs after plasmid-mediated expression in SFV-infected U4.4 cells (Blakqori et al., 2007). In the same study, it was reported that persistent infections of C6/36 cells with LACV resulted in the production of viral small RNAs (the exact size was not determined and these may correspond to piRNAs or other degradation products and not vsiRNAs since C6/36 cells were found later to be Dicer-2 deficient; see section "Dcr-2-Defective Ae. albopictus Cell Lines"). Furthermore, persistent infections of wild-type LACV and LACV deficient for NSs showed similar growth properties in C6/36 cells, indicating that NSs did not have a function as a suppressor of innate immunity (while a role as inhibitor of the exo-RNAi pathway could not be addressed in this experiment) (Blakqori et al., 2007). RVFV infection (another bunyavirus that encodes NSs) also did not interfere with RNAi silencing of a reporter gene, indicating the absence of VSR activity (Dietrich et al., 2017a).

\section{Other Mechanisms of Resistance Against RNAi}

Evidence has been presented for selection of genomes with mutations in regions highly targeted by RNAi as a mechanism of evasion (Brackney et al., 2009, 2015; review: Prasad et al., 2013; Blair and Olson, 2014, 2015a,b).

High targeting with vsiRNAs in regions of the genome of WNV could indeed be correlated with increased genetic diversity, indicating a mechanism for evading the RNAi response that restricts WNV replication (Brackney et al., 2009). Analysis of the RNAi response in mosquito bodies after feeding of an infectious blood meal of WNV also indicated differential modulation of viral small RNA profiles in mosquitoes of different Culex strains (Göertz et al., 2016), possibly implicating differences in selection pressure for particular virus-host combinations. Interestingly, no differences in the overall pattern of vsiRNAs between wild-type WNV and WNV defective for sfRNA1 (an abundant non-coding viral RNA produced during infection; see section "SfRNA") were observed, although specific vsiRNAs were identified that are derived from sfRNA1 in wild-type WNV (Göertz et al., 2016).

After passage of WNV in Drosophila S2 cells, the number of polymorphic sites was decreased after knock-down of $d c r$ 2 or ago-2 while it was increased after targeting the WNV genome with dsRNA (Brackney et al., 2015). RdRp enzymes from RNA viruses have a high error rate which could function as an evolutionary mechanism to escape targeting by vsiRNAs produced during the host antiviral response. Indeed, CHIKV viruses engineered with a high fidelity $\mathrm{RdRp}$ show lower infectivity and dissemination rate (Coffey et al., 2011), suggesting that generation of variety in viral genome sequences may be important for efficient infection (see also section "Engineering the RdRP Engines of Arbovirus Replication").

When the cell lines U4.4 from Ae. albopictus and Aag2 from Ae aegypti were used to analyze viral small RNA production following infection with SFV, accumulation of 21 nt vsiRNAs in hot spots and cold spots along the genome was observed but sequence analysis did not identify any correlation between predicted RNA secondary structure and abundance of vsiRNAs. Interestingly, hot spot-derived vsiRNAs were much less effective in triggering gene silencing as cold spot-derived viRNAs, indicating a possible mechanism of suppression of RNAi through a decoy mechanism (Siu et al., 2011). It has indeed been noticed that the propensity to produce siRNAs because of strong secondary structures can be accompanied by the resistance of those secondary structures to the RISC complex, leading to an evasion of the RNAi response (Fragkoudis et al., 2009).

\section{Inconclusive Evidence?}

While the existence of VSR genes in some arboviruses is suggested, it must be stressed that so far no conclusive proof was obtained that involves mutation of the VSR in the context of the 
arboviral genome during infections of natural hosts (discussed in O'Neal et al., 2014). Capsid proteins and many other proteins encoded by RNA viruses may have RNA binding activity that is unrelated to VSR activity in the context of viral infection but nevertheless may act as VSRs in other artificial assays, for instance during reporter assays in cell lines and biochemical assays of dicing and slicing.

Also the use of recombinant alphaviruses (SINV or SFV) to test candidate VSRs in infected mosquitoes may result in the identification of false positives. While recombinant SFV expressing capsid protein of ZIKV could replicate to higher levels than control SFV as observed before for recombinant SINV (Samuel et al., 2016), this effect was observed in both wildtype and Dcr-2-knock-out Aag-2 cells (Varjak et al., 2017a), indicating that the effect did not involve the antiviral siRNA (exo-RNAi) pathway. Consistent with this, it was observed that over-expression of capsid protein of ZIKV did not inhibit dsRNAor siRNA-mediated silencing of a reporter gene in Aag-2 cells (Varjak et al., 2017a). During ZIKV infections of Aag-2 cells, also no inhibition of dsRNA- or siRNA-mediated silencing of a reporter gene was detected (Varjak et al., 2017a).

\section{WHAT IS THE ROLE OF THE PIRNA PATHWAY IN ANTIVIRAL DEFENSE?}

In mosquitoes, Argonaute proteins of the PIWI class are expressed in somatic tissues, in contrast to Drosophila, where expression is predominantly in the germline (Lewis et al., 2016). Consistent with this observation, piRNAs of viral origin (viral piRNAs or vpiRNAs) were observed in somatic tissues during infections of mosquitoes (Morazzani et al., 2012). In addition, cell lines derived from mosquitoes were used to analyze the contribution of PIWI class proteins and production of vpiRNAs to the antiviral defense (Miesen et al., 2016b). Of significance was also the identification of mosquito cell lines that are defective in the production of vsiRNAs (Scott et al., 2010; Morazzani et al., 2012) which were subsequently employed to demonstrate the involvement of the piRNA pathway in antiviral defense. To directly assess the effect of the Dcr-2 deficiency in Aag2 cells, a clonal cell line defective in Dcr-2 was engineered using CRISPR-Cas and used to analyze the importance of the piRNA pathway versus the siRNA pathway in antiviral defense (Varjak et al., 2017b).

While piRNAs are defined by their association with Argonaute proteins of the PIWI class (for instance through their identification in specific immunoprecipitates), such information is not available in many studies that describe the generation of "viral small RNAs of piRNA size" or "vpiRNA-like small RNAs." Using a strict definition, typical hallmarks of piRNAs are: (1) sizes of 25-29 nt; (2) ping-pong amplification signature (U1 antisense, A10 sense); (3) enrichment of the separation of the $5^{\prime}$ ends of complementary viral piRNAs by $10 \mathrm{nt}$; and (4) resistance to $\beta$-elimination indicating $2^{\prime}$-O-methylation at the $3^{\prime}$ terminal nucleotide (Vodovar et al., 2012). However, it is also known that piRNAs that are generated in low abundance by the primary pathway (during early infection) will not show a ping-pong signature (Goic et al., 2016; see section "Alphaviruses"). The production of genuine piRNAs may also be obscured by the parallel generation of small RNAs through other degradation pathways. In some studies, "shoulders" of viral small RNAs of piRNA size are present in small RNA profiles that do not have a characteristic sequence logo (e.g., for profiles obtained from flavivirus-infected Culex mosquitoes; Fros et al., 2015; Göertz et al., 2016; and alpha virus-infected An. gambiae; Carissimo et al., 2015). Whether "viral small RNAs of piRNA size" or "vpiRNA-like small RNAs" in the absence of other characteristic features are produced by a similar piRNA pathway as that documented for the control of transposons (Senti and Brennecke, 2010), still requires further investigation.

\section{Production of VpiRNAs in Infected Mosquitoes}

Below follows a detailed analysis of the occurrence of the piRNA pathway as a potential antiviral defense mechanism in mosquitoes. Production of vpiRNAs is summarized in Table $\mathbf{1 .}$ An overview of the possible involvement of factors in the piRNA pathway is presented in Table 2 .

\section{Alphaviruses}

When Ae. aegypti and Ae. albopictus mosquitoes were injected with CHIKV, not only vsiRNAs (21 nt) were produced but also viral small RNAs of piRNA size (23-30 nt) (Morazzani et al., 2012). VpiRNAs exhibited a strong positive-strand bias and preferentially located on the region of the subgenomic RNA, with clear hotspots. Production of both vsiRNAs and vpiRNAs was also observed during oral infection of Ae. albopictus mosquitoes with CHIKV (Goic et al., 2016). During early infection (3 days p.i.), vpiRNA-like molecules (27-29 nt) were not abundant and did not show a sequence bias, in contrast to late infection (9 days p.i.), during which abundant vpiRNAs with ping-pong signature could be detected. The differences may reflect the production of primary vpiRNAs during early infection while abundant secondary vpiRNAs accumulate during late infection by the ping-pong amplification mechanism (Goic et al., 2016).

\section{Flaviviruses}

During oral infection of Ae. aegypti mosquitoes with DENV2 , the presence of piRNA-sized viral small RNAs (24-30 nt) was revealed preferentially during early infection along with the presence of canonical vsiRNAs and viral small RNAs of unusually short length (<20 nt) (Hess et al., 2011). After a decrease in viral small RNA levels at 4 days post infection, much higher levels of viral small RNAs were observed during late infection which corresponded mostly to vsiRNAs (20-23 nt). Viral small RNAs of piRNA size were preferentially of sense orientation and showed a weak signature of enrichment of adenine at the 10th base (A10) while no bias for the presence of uridine at position 1 (1U) was observed (Hess et al., 2011). It is noted that the preferential early accumulation of vpiRNAs during DENV-2 infection contrasts with their higher presence during later periods of infection by CHIKV (see above; Goic et al., 2016).

Similarly, after oral infection of Ae. albopictus mosquitoes with DENV-2, three classes of viral small RNAs could be identified: 
unusually small (ultrashort) RNAs (usRNAs; 13-19 nt), vsiRNAs (20-23 nt) and candidate vpiRNAs (24-30 nt) (Wang et al., 2018). Both vsiRNAs and piRNA-like small viral RNAs showed a strong positive-strand bias. A clear ping-pong signature was not detected, since the $1 \mathrm{U}$ bias for the antisense reads was absent while only a weak preference for A10 in the sense reads was observed; furthermore, no 10 nt overlap between sense and antisense reads was detected. Interestingly, differences in viral small RNA accumulation occurred in whole bodies and midguts of female adult mosquitoes: vsiRNAs were predominant in whole body samples while both candidate vpiRNAs and usRNAs were much more prevalent in midgut. In libraries from whole bodies of adult females, piRNA-like small viral RNAs derived mainly from a few hotspots in the DENV2 genome, located at the non-structural protein 5 (NS5) region, while a more broad distribution was observed for piRNA-like viral small RNAs across the DENV-2 genome in libraries of adult female midguts (Wang et al., 2018).

In Ae. albopictus, expression of piwi-1-4 is increased in adult females after blood feeding. However, no increase in expression of ago-3 and Piwi-related genes is observed following DENV-2 infection of adult females (Wang et al., 2018). During infection of Culex-derived cell lines with WNV, only production of vsiRNAs was observed and no induction in the expression of Piwi-related and ago-3 genes was detected (Rückert et al., 2019).

Besides the production of $21 \mathrm{nt}$ vsiRNAs, oral infection of $A e$. aegypti mosquitoes with ZIKV also resulted in the production of 25-30 nt viral small RNAs (Saldaña et al., 2017). However, because no ping-pong signature (U1, A10) was observed, the viral small RNAs, which were almost exclusively derived from the positive strand, may have been produced by other RNA degradation pathways and therefore could not be reliably identified as vpiRNAs.

\section{Bunyaviruses}

Rift Valley Fever virus infection (through infectious blood meal) of three different mosquito species (Ae. aegypti, Ae. vexans, Culex quinquefaciatus) resulted in the production of both vsiRNAs and vpiRNAs (Dietrich et al., 2017a). However, much lower levels of vsiRNAs and vpiRNAs were detected during infection of Culex mosquitoes. VpiRNAs of 26-30 nt that were detected displayed the characteristic ping-pong signature (sequence bias of $1 \mathrm{U}$ for genome strands and A10 for antigenome strands; 10 nt overlap between $5^{\prime}$-ends of complementary strands of small RNAs). Clear strand enrichment was observed for RVFV-derived vpiRNAs but differed according to the different segments ( $\mathrm{M}$ and $\mathrm{S}$ : antigenome bias; L: genome bias). For the $S$ segment, the majority of (sense, antigenome) vpiRNAs were derived from the region of the mRNA encoding the nucleocapsid $(\mathrm{N})$ protein (the other gene of the ambisense $\mathrm{S}$ segment (NSs) being transcribed from the antigenome strand; Dietrich et al., 2017a).

\section{vpiRNAs in Culex Mosquitoes}

The abundance of viral small RNAs following infection was observed to be much lower in Culex than in Aedes mosquitoes although this could be caused by infection status or virus-specific effects (Dietrich et al., 2017a). For Culex mosquitoes, low amounts of vpiRNAs were produced during infection with RVFV (Bunyavirales) (Dietrich et al., 2017a) while they were not reported during WNV or Usutu virus (USUV) (Flaviviridae) infection (Brackney et al., 2009; Fros et al., 2015; Göertz et al., 2016) or SINV (Alphavirus) infection (Miesen et al., 2016b). Although the relative importance of the contribution still needs to be investigated in detail, the detection of RVFV-specific vpiRNAs suggests that the possibility of contribution of the piRNA pathway to the antiviral defense can be extended to Culex mosquitoes.

\section{Analysis of the Role of the piRNA Pathway in Antiviral Defense in Mosquito Cell Lines}

Research on the significance of the piRNA pathway in antiviral defense has benefited from the availability of Ae. albopictus cell lines (C6/36 and C7-10) that are deficient in the production of vsiRNAs and that show higher production of viral titers in comparison with mosquito cell lines that are competent for vsiRNA production [Aag-2 (Ae. aegypti); U4.4 (Ae. albopictus)] (Scott et al., 2010; Morazzani et al., 2012). Another important line of research employed the Aag-2 cell line from Ae. aegypti in which relatively efficient knock-down of components of the piRNA- and siRNA-pathway could be achieved in order to evaluate their contribution to antiviral defense (Miesen et al., 2015). In these studies, production of vsiRNAs and vpiRNAs as well as the involvement of components of the siRNA and piRNA pathways are often directly compared.

Comparable results with respect to vsiRNA and vpiRNA production as in U4.4 and Aag-2 cell lines were also obtained with the TRA-171 cell line from the predatory mosquito Toxorhynchites amboinensis (Culicidae) infected with SFV (Togaviridae) (Donald et al., 2018). By contrast, no canonical vpiRNAs were observed after infection of the KC cell line of the midge $\mathrm{Cu}$. sonorensis with Bluetongue virus (Reoviridae), SBV or BUNV (both Bunyavirales) (Schnettler et al., 2013b; Dietrich et al., 2017b).

Below follows a detailed analysis of the occurrence of the piRNA pathway as a potential antiviral defense mechanism in mosquito cell lines. Comparison of the significance of the piRNA pathway with the exo-RNAi pathway regarding antiviral defense is provided. Production of vpiRNAs and vsiRNAs in cell lines is summarized in Table 3. An overview of the possible involvement of factors in the piRNA pathway (together with the exo-RNAi and miRNA pathways) in antiviral defense in the Aag- 2 cell line is presented in Table 4.

\section{Dcr-2-Defective Ae. albopictus Cell Lines}

During RNA virus infection of the C6/36 cell line from Ae. albopictus, non-canonical patterns of viral small RNAs are observed that are likely to be caused by its inability of dicing 
TABLE 3 | Viral small RNAs produced during arbovirus infections of mosquito cell lines.

\begin{tabular}{|c|c|c|c|}
\hline Virus & Cell line & Viral small RNAs & References \\
\hline \multicolumn{4}{|c|}{ Alphavirus } \\
\hline $\mathrm{CHIKV}$ & C6/36 (Ae. albopictus) C7/10 (Ae. albopictus) (both Dcr-2-defective) & 23-30 nt vpiRNA & Morazzani et al., 2012 \\
\hline $\mathrm{CHIKV}$ & C6/36 (Ae. albopictus) (Dcr-2-defective) & 25-30 nt vpiRNA & Goic et al., 2016 \\
\hline $\mathrm{CHIKV}$ & U4.4 (Ae. albopictus) & 21 nt vsiRNA 25-29 nt vpiRNA & Morazzani et al., 2012 \\
\hline SFV & Aag-2 (Ae. aegypti) & 21 nt vsiRNA 25-29 nt vpiRNA & Schnettler et al., 2013a \\
\hline SFV & AF5 clone (Aag-2) (Ae. aegypti) & 21 nt vsiRA 26-29 nt vpiRNA & Varjak et al., 2017b \\
\hline SFV & AF319 Dcr-2 KO (Aag-2) (Ae. aegypti) & 21 nt vsiRA 25-32 nt vpiRNA & Varjak et al., 2017b \\
\hline SFV & U4.4 (Ae. albopictus) & 21 nt vsiRNA 25-29 nt vpiRNA & Schnettler et al., 2013a \\
\hline SFV & TRA-171 (Toxorhynchites amboinensis) & 21 nt vsiRNA 26-29 nt vpiRNA & Donald et al., 2018 \\
\hline SINV & C6/36 (Ae. albopictus) (Dcr-2-defective) & 23-28 nt vpiRNA & Brackney et al., 2010 \\
\hline SINV & Aag-2 (Ae. aegypti) & 21 nt vsiRNA 25-29 nt vpiRNA & $\begin{array}{l}\text { Vodovar et al., } 2012 \\
\text { Miesen et al., } 2015\end{array}$ \\
\hline SINV & U4.4 (Ae. albopictus) & 21 nt vsiRNA 25-29 nt vpiRNA & Vodovar et al., 2012 \\
\hline \multicolumn{4}{|c|}{ Flavivirus } \\
\hline DENV & C6/36 (Ae. albopictus) (Dcr-2-defective) & 27 nt vpiRNA & Scott et al., 2010 \\
\hline DENV & Aag-2 & $21 \mathrm{nt}$ vsiRNA 25-30 nt vpiRNA & Miesen et al., $2016 a$ \\
\hline WNV & C6/36 (Ae. albopictus) (Dcr-2-defective) & 19-20 nt usRNA & Brackney et al., 2010 \\
\hline WNV & Hsu (Cx. quinquefasciatus) & 21 nt vsiRNA & Rückert et al., 2019 \\
\hline WNV & CT (Cx. tarsalis) & $21 \mathrm{nt}$ vsiRNA & Rückert et al., 2019 \\
\hline ZIKV & Aag-2 & 20-21 nt vsiRNA 25-28 nt vpiRNA & Varjak et al., 2017a \\
\hline \multicolumn{4}{|c|}{ Bunyaviridae } \\
\hline BUNV & Aag-2 (Ae. aegypti) & 14-19 nt usRNA 21 nt vsiRNA 25-28 nt vpiRNA & Dietrich et al., 2017b \\
\hline BUNV & U4.4 (Ae. albopictus) & 15-17 nt usRNA 21 nt vsiRNA 26-30 nt vpiRNA & Dietrich et al., 2017b \\
\hline BUNV & KC (Culicoides sonorensis) & 15-17 nt usRNA 21 nt vsiRNA 26-29 nt vpiRNA & Dietrich et al., 2017b \\
\hline LACV & C6/36 (Ae. albopictus) (Dcr-2-defective) & 24-28 nt vpiRNA & Brackney et al., 2010 \\
\hline LACV & C6/36 (Ae. albopictus) (Dcr-2-defective) & 25-29 nt vpiRNA & Vodovar et al., 2012 \\
\hline RVFV & C6/36 (Ae. albopictus) (Dcr-2-defective) & 14-19 nt usRNA 24-29 nt vpiRNA & Léger et al., 2013 \\
\hline RVFV & Aag-2 (Ae. aegypti) & 14-19 nt usRNA 21 nt vsiRNA 25-28 nt vpiRNA & Léger et al., 2013 \\
\hline RVFV & Aag-2 (Ae. aegypti) & 21 nt vsiRNA 24-32 nt vpiRNA & Dietrich et al., 2017a \\
\hline RVFV & U4.4 (Ae. albopictus) & 14-19 nt usRNA 21 nt vsiRNA 25-28 nt vpiRNA & Léger et al., 2013 \\
\hline SBV & Aag-2 (Ae. aegypti) & 15-17 nt usRNA 21 nt vsiRNA 25-30 nt vpiRNA & $\begin{array}{l}\text { Schnettler et al., 2013b } \\
\text { Dietrich et al., 2017b }\end{array}$ \\
\hline \multicolumn{4}{|c|}{ Reoviridae } \\
\hline BTV & Aag-2 (Ae. aegypti) & 21 nt vsiRNA 25-33 nt vpiRNA & Schnettler et al., 2013b \\
\hline
\end{tabular}

dsRNA substrates and a non-functional Dcr-2 enzyme (Scott et al., 2010). Genotyping revealed a homozygous frameshift mutation in the ORF of $d c r-2$ in C6/36 cells resulting in the formation of a premature stop codon (Morazzani et al., 2012). Another cell line of Ae. albopictus, C7-10, was also reported to be detective in the siRNA pathway, caused by a deletion of 33 AA between the DUF and PAZ domains of Dcr-2 (Morazzani et al., 2012). In several studies, the antiviral RNAi response in Dcr-2-deficient C6/36 and C7-10 cell lines was compared with the response in Dcr-2-competent cell lines such as Aag-2 (Ae. aegypti) and U4.4 (Ae. albopictus).

\section{Alphavirus}

During SINV infections of C6/36 cells mainly viral small RNAs of piRNA size (23-28 nt) were produced that were distributed unevenly between genomic (70\%) and antigenomic (30\%) strands (Brackney et al., 2010). Hot spots of piRNAsized small RNAs were observed in the subgenomic region that encodes the structural genes. Similarly, during infection of C6/36 and C7-10 cells with CHIKV (Alphavirus), only vpiRNAs of 23-30 nt size were detected that showed a clear ping-pong amplification (1U antisense, A10 sense) signature (Morazzani et al., 2012; Goic et al., 2016). By contrast, both vsiRNAs and vpiRNAs were produced during CHIKV infection in Dcr-2-competent U4.4 cells (Morazzani et al., 2012; Goic et al., 2016).

\section{Flavivirus}

During DENV-2 infections of C6/36 cells, viral small RNAs were generated that corresponded almost exclusively from the sense strand and were derived from a few specific regions of the genome (Scott et al., 2010). Moreover, the viral small RNAs had a size of $27 \mathrm{nt}$ and an enrichment of adenine at position 10 (A10) which indicates that they were generated by the piRNA pathway (Scott et al., 2010). On the other hand, infections with another flavivirus, WNV, resulted in the production of a high proportion of viral small RNAs of 17-18 nt that may correspond to degradation products by degradation pathways 
TABLE 4 | RNAi factors that affect arbovirus replication in Aag-2 (Ae. aegypti) cell line.

\begin{tabular}{|c|c|c|c|}
\hline Virus & RNAi factor & Phenotype after knock-down & References \\
\hline \multicolumn{4}{|c|}{ Alphavirus } \\
\hline $\mathrm{CHIKV}$ & Ago-2 & $\begin{array}{l}\text { Increased replication (replicon) } \\
\text { Increased viral replication }\end{array}$ & $\begin{array}{l}\text { McFarlane et al., } 2014 \\
\text { Varjak et al., } 2018 \mathrm{a}\end{array}$ \\
\hline $\mathrm{CHIKV}$ & Ago-3 & $\begin{array}{l}\text { Increased viral replication (minor effect) } \\
\text { Decreased production of vpiRNAs }\end{array}$ & Varjak et al., 2018a \\
\hline $\mathrm{CHIKV}$ & Piwi-4 & Increased viral replication & Varjak et al., 2018a \\
\hline $\mathrm{CHIKV}$ & Piwi-5 & Decreased production of vpiRNAs & Varjak et al., 2018a \\
\hline $\mathrm{CHIKV}$ & SpnE & $\begin{array}{l}\text { Increased viral replication } \\
\text { Decreased production of vpiRNAs (minor effect) }\end{array}$ & Varjak et al., 2018a \\
\hline SFV & Ago-2 & Increased viral replication & $\begin{array}{l}\text { Schnettler et al., 2013a; } \\
\text { Varjak et al., } 2017 \text { a }\end{array}$ \\
\hline SFV & Ago-2 & $\begin{array}{l}\text { Increased viral replication in AF5 (Dcr-2 competent) } \\
\text { More pronounced than in AF319 (Dcr-2 defective) }\end{array}$ & Varjak et al., 2017b \\
\hline SFV & Ago-3 & Increased viral replication (minor effect) & Varjak et al., 2017a \\
\hline SFV & Ago-3 & Decreased production of vpiRNAs & Varjak et al., 2018a \\
\hline SFV & all Piwi/Ago-3 & No production of vpiRNAs & Schnettler et al., $2013 a$ \\
\hline SFV & Piwi-4 & Increased viral replication & $\begin{array}{l}\text { Schnettler et al., 2013a; } \\
\text { Varjak et al., 2017a, 2018a }\end{array}$ \\
\hline SFV & Piwi-4 & $\begin{array}{l}\text { Increased viral replication with similar efficiency in both AF5(Dcr-2 competent) and } \\
\text { AF319 (Dcr-2 defective) }\end{array}$ & Varjak et al., 2017b \\
\hline SFV & Piwi-5 & Decreased production of vpiRNAs & Varjak et al., 2018a \\
\hline SFV & Piwi-6 & Decreased production of vpiRNAs (minor effect) & Varjak et al., 2018a \\
\hline SFV & SpnE & $\begin{array}{l}\text { Increased viral replication } \\
\text { Decreased production of vpiRNAs (minor effect) }\end{array}$ & Varjak et al., 2018a \\
\hline SINV & Piwi-4 & $\begin{array}{l}\text { Decreased production of vpiRNAs (minor effect) } \\
\text { Increased production of vsiRNAs }\end{array}$ & Miesen et al., 2015 \\
\hline SINV & Piwi-5 & $\begin{array}{l}\text { Decreased production of vpiRNAs } \\
\text { Increased production of vsiRNAs }\end{array}$ & Miesen et al., 2015 \\
\hline SINV & Ago-3 & Decreased production of vpiRNAs & Miesen et al., 2015 \\
\hline \multicolumn{4}{|c|}{ Flavivirus } \\
\hline DENV & Ago-3 & Decreased production of vpiRNAs & Miesen et al., 2016a \\
\hline DENV & Piwi-5 & Decreased production of vpiRNAs & Miesen et al., 2016a \\
\hline DENV & Piwi-6 & Decreased production of vpiRNAs (minor effect) & Miesen et al., 2016a \\
\hline ZIKV & Dcr-2 & Increased viral replication & Varjak et al., 2017a \\
\hline ZIKV & Ago-2 & Decreased viral replication (proviral) & Varjak et al., 2017a \\
\hline ZIKV & Ago-3 & Decreased viral replication (proviral) & Varjak et al., 2017a \\
\hline ZIKV & Piwi-4 & Increased viral replication & Varjak et al., 2017a \\
\hline \multicolumn{4}{|c|}{ Bunyaviridae } \\
\hline BUNV & Ago-1 & Decreased viral replication (proviral) & Dietrich et al., 2017b \\
\hline BUNV & Ago-2 & Increased viral replication & Dietrich et al., 2017b \\
\hline BUNV & Ago-3 & Decreased viral replication (proviral) & Dietrich et al., 2017b \\
\hline BUNV & Piwi-4 & Increased viral replication & Dietrich et al., 2017b \\
\hline BUNV & Piwi-6 & Decreased viral replication (proviral) & Dietrich et al., 2017b \\
\hline CW & Ago-1 & Decreased viral replication (proviral) & Dietrich et al., 2017b \\
\hline CW & Ago-2 & Increased viral replication & Dietrich et al., 2017b \\
\hline CW & Piwi-4 & Increased viral replication & Dietrich et al., 2017b \\
\hline SATV & Ago-1 & Increased viral replication & Dietrich et al., 2017b \\
\hline SATV & Ago-2 & Increased viral replication & Dietrich et al., 2017b \\
\hline SBV & Ago-1 & Increased viral replication & Dietrich et al., 2017b \\
\hline SBV & Ago-2 & Increased viral replication & Dietrich et al., 2017b \\
\hline SBV & Piwi-5 & Decreased viral replication (proviral) & Dietrich et al., 2017b \\
\hline RVFV & Ago-2 & Increased viral replication & Dietrich et al., $2017 a$ \\
\hline RVFV & Ago-3 & Increased viral replication & Dietrich et al., 2017a \\
\hline RVFV & Piwi-4 & Increased viral replication & Dietrich et al., $2017 \mathrm{a}$ \\
\hline
\end{tabular}

that are not related to RNAi. The vast majority of viral small RNAs during WNV infection of C6/36 cells were of sense polarity while no viral small RNAs of piRNA size were detected (Brackney et al., 2010).

\section{Bunyavirales}

Analysis of viral small RNAs produced during infections of C6/36 cells with LACV also revealed the predominant presence of viral small RNAs of piRNA-like size (24-28 nt) (Brackney et al., 2010; 
Vodovar et al., 2012). Despite LACV being a virus with a segmented negative-sense RNA genome, viral small RNAs from the positive-sense strand (also corresponding to the mRNA) were predominant $(>70 \%)$ although differences among the three segments were observed (Vodovar et al., 2012). The S segment was much more targeted (by 10-fold) than the $M$ and $\mathrm{L}$ segments which can be correlated with the much higher abundance of the $S$ segment mRNA compared to the mRNAs of the other segments (Brackney et al., 2010). LACV-derived small RNAs in C6/36 cells are clearly produced by the piRNA pathway since they show a ping-pong amplification signature (U1 antisense, A10 sense) and the $5^{\prime}$-ends of complementary small RNAs are preferentially separated by $10 \mathrm{nt}$ (Vodovar et al., 2012). Regarding the biogenesis of LACV-derived piRNAs, it can be hypothesized that abundant mRNAs are initially targeted to produce primary (sense) piRNAs, which will subsequently target antisense genomic strands. Viral piRNA production from the genomic strands, however, will be limited because of their low abundance. On the other hand, the few vpiRNAs of antisense orientation from the second step presumably can generate abundant vpiRNAs from the abundant positive strand RNAs (i.e., mRNAs) that are present in the infected cells (Vodovar et al., 2012).

A comparative study was carried out among Dicer-2competent U4.4 and Aag-2 cell lines and the Dcr-2-defective C6/36 cell line with respect to RVFV infection (ZH548 strain; Léger et al., 2013). Interestingly, persistent infections were only obtained with U4.4 and Aag-2 cells which could be correlated with the clearance of viral NSs filaments from the nuclei of the infected cells. In infections of mammalian cells, the nonstructural NSs protein also forms nuclear filaments and plays a fundamental role in the mechanism of pathogenicity, i.e., by interference with cellular transcription. VpiRNAs (27-28 nt) with ping-pong signature were observed after RVFV infection of C6/36 cells, besides the presence of $24-25 \mathrm{nt}$ and $21 \mathrm{nt}$ viral small RNAs that did not show a ping-pong signature. Characteristic of RVFV infections of C6/36 cells (but also observed in Aag2 and U4.4 cells) was also the production of highly abundant very small viral RNAs (14-19 nt) that are probably generated by another degradation pathway, a phenomenon also observed during WNV infection of C6/36 cells (Brackney et al., 2010; see section "Flavivirus"). While persistent infections of C6/36 cells with RVFV were not obtained (infected C6/36 cells could not be passaged), it was nevertheless observed that infection with a particular RVFV strain could protect against secondary infections ("super-infections") of a different strain of RVFV in this cell line. These data were interpreted to suggest that functional Dcr-2 is not required for pathogen-derived resistance and that the piRNA pathway alone can support some antiviral response.

\section{Knock-Down of Components of piRNA Pathway in Aag-2 Cells}

Aag-2, an Ae. aegypti cell line of embryonic origin, has been used recently more frequently as a model for immunity studies representative of mosquitoes (Barletta et al., 2012). Besides the genes of the miRNA and siRNA pathways, Aag-2 cells also express the PIWI class Argonaute proteins that are present in somatic tissues of adult mosquitoes (piwi-4, piwi-5, piwi-6 and ago-3; but not piwi-1-3 or piwi-7; Miesen et al., 2015). However, Aag-2 cells are also persistently infected with the mosquitospecific viruses Phasi-Charoen Like virus (PCLV; Bunyavirales) and cell-fusing agent virus (CFAV; Flaviviridae) (Weger-Lucarelli et al., 2018) (see also section "RNAi and the Antiviral Defense Against Mosquito-Specific Viruses"), that may affect arbovirus infections in this cell line (Zhang et al., 2017; Schultz et al., 2018). The impact of mosquito-specific virus infections to modulate concomitant arbovirus infections in mosquitoes is an active area of research (Fredericks et al., 2019).

\section{Alphavirus}

Infections of Aag-2 cells with alphavirus (SINV, SFV, CHIKV) did not only result in production of vsiRNAs but also of viral small RNAs that have the characteristics of piRNAs (Morazzani et al., 2012; Vodovar et al., 2012; Schnettler et al., 2013a; Miesen et al., 2015). VpiRNAs were mainly of sense orientation, were mostly derived from regions at the subgenomic RNA and showed the distinctive signs of ping-pong amplification (U1 antisense and A10 sense; $10 \mathrm{nt}$ overlap between $5^{\prime}$-termini of complementary reads).

Knock-down of individual Piwi genes established the involvement of cytoplasmic Piwi-5 and Ago-3 in the biogenesis of SINV-derived vpiRNAs in Aag-2 cells. Sequencing of vpiRNAs in immunoprecipitates reveals association of sense vpiRNAs of A10 bias with Ago-3, while antisense vpiRNAs of U1 logo are preferentially bound to Piwi-5 and Piwi-6 (Miesen et al., 2015). On the other hand, Piwi-4 was not found to be associated with any vpiRNAs. Although knock-down of piwi-4 affected accumulation of piRNAs derived from transposable elements, no piRNAs corresponding to transposon sequences were detected in Piwi-4 immunoprecipitates, leading to the proposal that Piwi-4 indirectly regulates the function of the piRNA pathway (i.e., in the absence of direct binding of piRNAs), possibly by modulating the activity of the other piRNA factors or regulating the amount of substrate that enters the piRNA pathway. The diversification of Piwi-related factors in Ae. aegypti mosquitoes likely reflects their specialization in the control of different classes of parasitic nucleic acids (mainly transposons), with Piwi-5 and Ago-3 involved more specifically in production of piRNAs during viral infections (Miesen et al., 2015).

Knock-down experiments of Piwi-related genes in Aag-2 cells also revealed increased SFV replication following silencing of piwi-4 (as well as ago-2). Knock-down of all expressed Piwirelated proteins (piwi-2, piwi-3, piwi-4, piwi-5, piwi-6, piwi-7 and ago-3) resulted in a strong decrease in production of vpiRNAs during SFV infection. However, knock-down of piwi-4 had no effect on vpiRNA accumulation, indicating an effector function of Piwi-4 to target and silence the virus independent of vpiRNAs (Schnettler et al., 2013a).

A clonal cell line derived from Aag-2 cells (AF5) was used to engineer a Dcr-2-defective cell line (AF319) representative of Ae. aegypti by CRISPR-Cas9 mediated engineering (Varjak et al., 2017b). When infected with SFV, production of vsiRNAs was abolished in AF319 cells while the levels of vpiRNAs were greatly enhanced which correlated with increased viral 
replication compared to AF5 cells (as also observed for Dicerdefective C6/36 and C7/10 cells derived from Ae. albopictus). Despite differences in vpiRNA levels, the properties of SFVderived vpiRNAs were very similar between Dcr-2-deficient AF319 and Dcr-2-competent AF5 cells such as distribution along the viral genome and the occurrence of the ping-pong signature. Re-introduction of Dcr-2 by expression plasmids also did not alter the properties of vpiRNAs in AF319 cells (Varjak et al., 2017b). Knock-down of ago-2 had a much reduced effect on SFV replication in AF319 cells, in comparison with AF5 cells, confirming the dependence of the antiviral effects of Ago-2 on production of vsiRNAs produced by Dcr2. On the other hand, silencing of piwi-4 increased viral replication in both AF5 and AF319 cells, indicating that the antiviral effect of Piwi-4 does not require Dcr-2. In pulldown experiments using extracts from Dcr-2-deficient AF319 cells (infected with SFV), vpiRNAs with ping-pong signature (but without positive strand bias as is commonly observed) were detected in association with both Ago-2 and Piwi-4. However, binding may be indirect since Piwi-4 is found in complexes with proteins of both siRNA (Ago-2, Dcr-2) and piRNA (Piwi-5, Piwi-6, Ago-3) pathways (Varjak et al., 2017b; see also section "Possible Cross-Talk Between siRNA and piRNA Pathway?"). Furthermore, silencing of piwi-5 or ago-3 (both previously shown to be involved in vpiRNA production in Aag-2 cells; Miesen et al., 2015, 2016a) in AF319 cells also did not result in increased SFV replication. The experiments indicate strongly that Piwi-4 is an antiviral effector that is independent of vsiRNA and vpiRNA production. On the other hand, vpiRNAs do not seem to play an antiviral role since they seem to be incapable to replace the role of vsiRNAs in Dcr-2deficient cells.

Following the demonstration that knock-down of ago-2 resulted in increased expression of a CHIKV reporter replicon (McFarlane et al., 2014), similar knock-down studies were carried out for ago-3 and Piwi-related genes which showed a clear antiviral effect for piwi-4 while the effect for ago-3 was minor (Varjak et al., 2018a). No antiviral role was revealed for piwi-5 or piwi-6. As for SINV and SFV, knock-down of ago-3 and piwi-5 and, to a lesser extent, piwi-6, resulted in decreased vpiRNA levels during CHIKV infection.

Knock-down of other factors of the piRNA pathway revealed the antiviral activity of the helicase Spindle-E (SpnE), that was identified as a cofactor in the ping-pong amplification loop in Drosophila (Varjak et al., 2018a). No antiviral role was uncovered during silencing of other potential cofactors in the piRNA pathway of mosquitoes (Qin, Vasa, Zucchini, Armitage, GasZ, Hen1 and factors involved in heterochromatin silencing). Knock-down of SpnE also resulted in partial suppression of vpiRNAs, similar to knock-down of piwi-6. However, the antiviral effect of SpnE could not be correlated with the production of both vsiRNAs and vpiRNAs and therefore may be related to its more general role in the regulation of RNA metabolism. Interestingly, the antiviral role of $\operatorname{SpnE}$ seems to be limited to alphaviruses (SFV, CHIKV) since its knock-down did not affect BUNV (Bunyavirales) or ZIKV (Flaviviridae) replication (Varjak et al., 2018a).

\section{Flavivirus}

During DENV-2 infection of Aag-2 cells, abundant vpiRNAs were also detected in addition to the production of vsiRNAs (Miesen et al., 2016a). DENV-derived vpiRNAs in Aag-2 cells have a characteristic size of $25-30 \mathrm{nt}$, are resistant to $\beta$-elimination (and therefore likely $2^{\prime}$-O-methylated at their $3^{\prime}$ termini), show an almost exclusively sense orientation and derive from a very limited number of hotspots ( $85 \%$ of reads correspond to just four vpiRNA sequences; Miesen et al., 2016a). Gene silencing studies in Aag- 2 cells reveals the dependence of DENV-derived piRNAs on ago-3, piwi-5 and (partially) piwi-6. However, none of the knock-downs of ago-3 and Piwi-related genes (as well as ago-2) resulted in an increase in DENV replication levels in Aag-2 cells (Miesen et al., 2016a).

Infection of Aag-2 cells with ZIKV resulted in production of both vsiRNAs and vpiRNAs (Varjak et al., 2017a). VpiRNAlike molecules (25-29 nt) were derived from a hotspot in the NS5 region at a similar position of the subgenomic RNA as observed during DENV infections. However, no pingpong signature was observed in the piRNA-like viral small RNAs. Immunoprecipitation experiments showed association of vsiRNAs with Ago-2 and the association of vpiRNA-like molecules with Ago-3 but not Piwi-5 or Piwi-6 during ZIKV infections (Varjak et al., 2017a). In immunoprecipitates of Piwi4, relatively small amounts of both vsiRNAs and vpiRNA-like small RNAs were detected (but see also section "Possible CrossTalk Between siRNA and piRNA Pathway?"). Silencing of piwi-4 resulted in an increase of replication of ZIKV reporter virus in Aag-2 cells, in contrast to silencing of piwi-5 and piwi6. Possible proviral functions of ago-2 and ago-3 were also revealed during infections with ZIKV in Aag-2 cells (Varjak et al., 2017a). Interestingly, an antiviral function of $d c r-2$ could be demonstrated during ZIKV infection, in contrast to ago-2.

\section{Bunyavirales}

With respect to the accumulation of viral small RNAs during RVFV infection, it was observed that during the early phases of acute infection, vsiRNAs predominate in Aag-2 and U4.4 cells while during later phases of acute infection and during persistent infection, vpiRNAs became more important (Léger et al., 2013; also observed during alphavirus infection; Goic et al., 2016). While vsiRNAs mapped with roughly equal proportion to genomic and antigenomic strands for the $\mathrm{L}$ and $\mathrm{M}$ segments, a strong bias for the antigenomic (coding) strand was observed for the vpiRNAs, especially for the S segment, which is known to produce abundant mRNAs. VpiRNAs showed a clear pingpong signature, with enrichment for $\mathrm{U} 1$ and $\mathrm{A} 10$ on the genomic (antisense) and antigenomic (sense) strands, respectively. Pingpong signature was more evident for $27-28 \mathrm{nt}$ than for $24-25 \mathrm{nt}$ viral small RNAs but could also be detected in $21 \mathrm{nt}$ reads during persistent infections of Aag-2 cells.

Viral small RNAs produced during RVFV infection of Aag-2 cells were functional since silencing of reporter constructs with RVFV target sequences was observed during RVFV infection of Aag- 2 cells. RVFV could also be targeted by the RNAi machinery after transfection of dsRNA targeting the $\mathrm{S}$ and $\mathrm{L}$ segments. Furthermore, enhanced RVFV replication was observed after 
silencing of ago-2, piwi-4 and ago-3 (but not piwi-5 or piwi-6) in Aag- 2 cells, implicating the involvement of both the siRNA and the piRNA pathway in antiviral defense (Dietrich et al., 2017a).

Also infections of SBV (with Culicoides midges as natural vectors/hosts) caused production of both vsiRNAs and vpiRNAs in non-host Aag-2 cells (Schnettler et al., 2013b). As for LACV and RVFV, vsiRNAs were equally presented between genomic and antigenomic segments while vpiRNAs were mainly derived from the antigenomic (sense) strand of the $S$ segment. In addition, viral small RNAs of very small size (15-17 nt) were produced, mainly from segments $M$ and L. In infected Aag-2 cells, SBV-derived small RNAs of sizes 24-30 nt displayed characteristics of piRNAs such as a ping-pong signature (U1 antisense, A10 sense) and the preferential separation of $5^{\prime}$-termini of complementary reads by 10 nt (Schnettler et al., 2013b).

Infections of Aag- 2 cells (and U4.4 cells) with BUNV resulted in production of both vsiRNAs and vpiRNAs (Dietrich et al., 2017b). VsiRNAs of 21 nt length were predominant for the $\mathrm{L}$ segment and mapped to both genome and antigenome in a hot spot and cold spot pattern. VpiRNAs of 24-30 nt length were the major species for the $\mathrm{M}$ and $\mathrm{S}$ segments with a bias for the genome.

Silencing of different Argonaute and Piwi-related genes in Aag-2 cells revealed different genetic requirements for antiviral defense against mosquito-borne [BUNV and Cache Valley virus $(\mathrm{CVV})]$ and midge-borne bunyaviruses [SBV and Sathuperi virus (SATV)]. While ago-2 provided antiviral activity to all infections, piwi-4 only protected against mosquito-borne bunyaviruses (Dietrich et al., 2017b). Interestingly, proviral functions of ago-3 and piwi-6 were revealed for infections with mosquitospecific BUNV and of piwi-5 for (midge-specific) SBV infections. The analysis therefore supports the adaptation of the piRNA pathway in Aag-2 cells to specific vector-virus combinations. The miRNA pathway also has a proviral function during infections with mosquito-specific bunyaviruses, while silencing of ago-1 increases replication of midge-specific viruses (antiviral function; Dietrich et al., 2017b).

\section{Possible Cross-Talk Between siRNA and piRNA Pathway?}

There are several indications of interactions between siRNA and piRNA pathways during antiviral defense in mosquitoes which were revealed in experiments with recombinant viruses expressing the B2 VSR that sequesters dsRNA and also by the detection of protein complexes that contain factors of both RNAi pathways and their respective small RNAs. During viral infection temporal patterns of vpiRNA and vsiRNA accumulation were also observed suggesting the predominance of a particular pathway at different stages of infection.

Infection of $\mathrm{Ae}$. albopictus mosquitoes with recombinant $\mathrm{CHIKV}$ that expresses the B2 VSR protein (CHIKV-B2) resulted in higher virulence/mortality and decreased production of vsiRNAs while levels of vpiRNAs were increased (Morazzani et al., 2012; see also section "Evidence for Presence of VSR Genes in Arboviral Genomes" for discussion of VSRs). The capacity of the dsRNA-binding protein B2 to influence both
vsiRNA and vpiRNA pathways may indicate that both pathways are initially activated by viral dsRNAs acting as pathogenassociated molecular patterns (PAMPs). In Dcr-2-defective C6/36 cells, infection by CHIKV-B2 caused higher virulence, decreased production of vpiRNAs and a relative increase in the number of viral genomes compared to wild-type CHIKV or CHIKV expressing mutant B2 (Morazzani et al., 2012). The involvement of the dsRNA-binding protein B2 again suggested the possibility that dsRNA plays a role as a PAMP to activate the antiviral piRNA pathway in Dcr2-defective Ae. albopictus cell lines (Morazzani et al., 2012). However, low levels of vsiRNAs are also produced during CHIKV infection of C6/36 cells (Goic et al., 2016) by an unknown mechanism (e.g., persistent low activity of Dcr2 or ectopic activity of Dcr-1) and B2 could therefore act through further inhibition of vsiRNA production. Furthermore, transfection of luciferase dsRNA can inhibit replication of the corresponding reporter replicon of WNV (Pitaluga et al., 2008), indicating the presence of a partially active exo-RNAi pathway in C6/36 cells. As previously mentioned, other data, using Dcr2-deficient Aag-2 cells, indicate the independence of the piRNA pathway from the antiviral siRNA pathway and question the role of vpiRNAs during antiviral defense (Varjak et al., 2017b). When dsRNA is transfected into Aag-2 and U4.4 cells, $21 \mathrm{nt}$ siRNAs but no vpiRNAs are produced, providing further evidence that dsRNA preferentially/exclusively activates the dsRNA pathway (Schnettler et al., 2013a).

During infection of Aag-2 cells by alphavirus, the presence of both vsiRNAs and vpiRNAs was revealed in Piwi-4 immunoprecipitates although the levels were rather low (Varjak et al., 2017b). For both SINV and SFV infections, vpiRNAs with ping-pong characteristics were also found in immunoprecipitates of Ago-2. In addition, it was demonstrated that Piwi-4 can exist in complexes with other proteins of both siRNA (Ago2, Dcr-2) and piRNA (Piwi-5, Piwi-6, Ago-3) pathways. It is therefore unknown whether viral small RNAs detected in Piwi-4immunoprecipitates correspond to direct binding to Piwi-4 or to binding to other interacting proteins (Varjak et al., 2017b). While these data point to physical interactions between piRNA- and siRNA-associated Argonaute proteins during antiviral defense, its functional significance remains to be elucidated.

In several cases, differential temporal accumulation of vsiRNAs and vpiRNAs during arboviral infection was reported. Moreover, the preferential generation of vsiRNAs versus vpiRNAs and vice versa over time may depend on the virus and the infection system used (systemic infection of cell lines versus oral infection of mosquitoes). During oral infection of Ae. aegypti with DENV, vpiRNAs predominate during early infection while vsiRNAs become much more abundant during late infection (Hess et al., 2011). In Aag-2 and U4.4 cells that were infected by RVFV, by contrast, early stages of acute infection are mainly associated with vsiRNA production and late stages of acute infection and persistent infections with vpiRNAs (Léger et al., 2013). Also during oral infection of Ae. albopictus with CHIKV, vpiRNAs became abundant during late stages with characteristic features such as ping-pong signature and the association with predominant hotspots on the genome (Goic et al., 2016). Production of vsiRNAs likely correlates with 
activation of antiviral defense given the demonstrated antiviral role of the exo-RNAi pathway. On the other hand, in the absence of a clearly demonstrated antiviral role, vpiRNAs may be generated as secondary products when viral replication expands in the infected cells and tissues.

\section{Expansion of piRNA Genes and Transposon Control}

In Aag-2 cells, derived from Ae. aegypti, abundant small RNAs were detected that mapped to transposon sequences (Vodovar et al., 2012; Miesen et al., 2015). Most endo-siRNAs were associated with DNA transposons such as miniature inverted terminal elements (MITEs) (Arensburger et al., 2011); piRNAs mainly derived from retrotransposons and showed an antisense bias and ping-pong signature (Vodovar et al., 2012; Miesen et al., 2015). Knock-down experiments established that both piwi-4 and piwi-5 were required for production of antisense transposonderived piRNAs while production of sense piRNAs depended on ago-3. Consistent with this, immunoprecipitates of Piwi5 and Ago-3 were enriched for transposon-derived piRNAs of antisense and sense orientation, respectively. On the other hand, Piwi-4 was relatively depleted for transposon-derived piRNAs which was also observed for vpiRNAs (Miesen et al., 2015). Interestingly, retrotransposons could be classified into different groups based on the dependence of their abundance upon knockdown of particular Piwi-related genes (piwi-4, piwi-5, piwi-6, ago-3) and on their association with particular Piwi proteins (Piwi-5, Piwi-6, Ago-3) in immunoprecipitates. Since the genome of Ae. aegypti is very rich in transposon sequences (Nene et al., 2007; Arensburger et al., 2011), it can be speculated that the diversification of its piRNA pathway occurred from the need to control parasitic RNAs from different origins. During this process, Piwi-5 and Ago-3 became the main factors for the production of vpiRNAs during viral infections even if the role in antiviral defense remains unclear. The great majority of vpiRNAs during occurring infections are of sense orientation and therefore must be derived from viral genomes of positive sense RNA viruses and viral mRNAs but it is currently unknown how these viral precursors are differentiated from abundant cellular mRNAs and can act as sources for piRNAs (Miesen et al., 2015).

However, despite the abundance of transposon sequences in its genome, only $19 \%$ of piRNAs map to transposon sequences in Ae. aegypti, in contrast to Drosophila, where the proportion is much higher (51\%) (Arensburger et al., 2011). Recently, a broader role for piRNAs and PIWI class proteins in the regulation of cellular gene expression in both soma and germline became more apparent in different organisms (Robine et al., 2009; Ishizu et al., 2015; Lewis et al., 2018). In Aag-2 cells, a significant proportion of piRNAs are produced from transcripts of protein-coding genes according to a similar mechanism as for the production of vpiRNAs during arbovirus infection (Girardi et al., 2017). As is the case for transposons (Miesen et al., 2016a), cellular transcripts can be grouped in different classes with respect to production of vpiRNAs, i.e., according to the dependence/association with particular Piwirelated genes/proteins and ago-3/Ago-3. Differentiation of the
piRNA pathway in Ae. aegypti therefore may not only reflect strategies of transposon control but also modes of regulation of gene expression by piRNAs. Interestingly, protein-coding genes can produce piRNAs via a ping-pong mechanism that involves piwi-5 and ago-3 as is observed for the production of vpiRNAs (Girardi et al., 2017). It can therefore be asked whether viral (anti)genome strands or mRNAs are recruited to this mechanism by chance or whether it could reflect a still unknown regulatory mechanism.

\section{AMPLIFICATION OF THE ANTIVIRAL RNAi RESPONSE AND POTENTIAL ESTABLISHMENT OF IMMUNE MEMORY}

\section{Arboviral DNA Forms}

During arboviral (CHIKV, DENV, WNV, LACV) infections of mosquitoes and/or mosquito cell lines, viral DNA forms (vDNAs) are produced by cellular retrotransposon-derived reverse transcriptase (RT) activity (Goic et al., 2016; Nag et al., 2016; Rückert et al., 2019). Synthesis of vDNA was observed to stimulate vsiRNA production especially during the early stages of oral infection of Ae. albopictus mosquitoes by CHIKV. While inhibition of RT activity increased mortality in infected mosquitoes, viral replication levels remained unaffected leading to the proposal that production of vDNA forms increased the tolerance (but not the resistance) of mosquitoes to CHIKV infections (Goic et al., 2016). During infection of Ae. aegypti mosquitoes with CHIKV, both linear and circular vDNA forms are produced (Poirier et al., 2018). In extension to studies with Drosophila, procedures that increase the production of vDNA forms, such as the increased presence of defective viral genomes (DVGs) in the inoculum, could decrease the infection rate of CHIKV during blood feeding of Ae. aegypti mosquitoes (Poirier et al., 2018).

Experiments with mosquito cell lines (Aag, C6/36 and Culexderived cell lines) showed that production of vDNA forms did not involve the entire viral genome but rather discrete patches that indicate separate events of template switching of RT activity between template retrotransposon RNA and nearby arboviral genomic RNA or mRNA (Nag et al., 2016; Rückert et al., 2019). Production of vDNA was dependent on the abundance of arboviral genomes and mRNAs since it was found to be enhanced in C6/36 cells where viral titers are typically much higher because of a defect in the exo-RNAi pathway (Morazzani et al., 2012).

\section{Arboviral Sequences Integrated in Mosquito Genomes}

While synthesis of vDNA forms may play a role in antiviral defense during occurring viral infections, sequencing of the genomes of several mosquito species has also established that sequences of RNA viruses can become integrated in the host genomes and possibly maintained as reservoirs of acquired immune memory (Katzourakis and Gifford, 2010; Arensburger et al., 2011; Girardi et al., 2017). Inserted viral sequences are generally known as "endogenous viral elements" (EVEs) but of 
interest here are "non-retroviral integrated RNA virus sequences" (NIRVS) that correspond to insertions of RNA viruses that lack an RT gene and that are thought to represent very rare events (Ballinger et al., 2014). NIRVS are sparse in the genomes of $C x$. quinquefasciatus and anopheline species but 10-fold more frequent in Ae. aegypti and Ae. albopictus; their abundance therefore does not seem to correlate with viral exposure (high in both Culex and both Aedes species) but may be the result of a combination of the expansion of the piRNA pathway (in Culex and both Aedes species but not in Anopheles) and the load of retrotransposons in the genome (high in both Aedes species; intermediate in Culex; low in Anopheles) (Chen et al., 2015; Palatini et al., 2017). Infection intensity may be an important factor since it would stimulate the formation of vDNA (Olson and Bonizzoni, 2017).

Mechanisms proposed for integration include hitchhiking on retrotransposable elements and via double-stranded break repair (Liu et al., 2010; Fort et al., 2012). A close association of NIRVS and transposable elements is observed in Aedes mosquitoes, particularly with long terminal repeat (LTR) retrotransposons of the Gypsy and Pao Bell families (Palatini et al., 2017). The most abundant NIRVS derive from the Flavivirus genus and Rhabdoviridae family (Crochu et al., 2004; Fort et al., 2012; Pischedda et al., 2019) with Bunyavirales- and Reoviridae-like sequences much more rare (and alphavirus-derived sequences absent; Palatini et al., 2017). Most NIRVS correspond to mosquito-specific viruses that are related to arboviruses (i.e., they do not cycle with vertebrate hosts) but it should be considered that mosquito-specific viruses are more likely to be transmitted vertically through the germline and that only NIRVS integrated in the germline will be passed to the next generation. The occurrence of arboviral NIRVS in genomes of somatic cells would not be passed to the next generation and more difficult to detect.

Interestingly, NIRVS are located in regions of the Aedes genome that preferentially produce piRNAs (Arensburger et al., 2011; Girardi et al., 2017; Palatini et al., 2017) and recently were more precisely designated in Aag- 2 cells to loci that resemble piRNA clusters from Drosophila (Whitfield et al., 2017). NIRVSspecific piRNAs were biased for $U$ at the first position and preferentially of antisense orientation, indicating the potential to interfere with invading viral genomes or mRNAs; for NIRVS derived from rhabdoviruses, a ping-pong signature was also observed. Knock-down and immunoprecipitation experiments in Aag-2 cells established dependence for their formation on piwi-4, piwi-5, and piwi-6 and their association with Piwi-5 and Piwi-6 proteins (Palatini et al., 2017). A direct interaction between Phasi Charoen-like virus (PCLV), a bunyavirus that persistently infects Aag-2 cells, and NIRVS-derived (antisense) piRNAs from a piRNA cluster was also demonstrated. VpiRNAs of sense orientation are produced from different regions of the PCLV genome but, in a region of common sequences between NIRVS and the virus, a peak of sense piRNA production from the virus was separated by 10 bp from a peak of antisense piRNAs apparently derived from the NIRVS, an interaction which is consistent with the ping-pong mechanism (Whitfield et al., 2017). This observation indicates the possibility that NIRVS-derived
piRNAs contribute to the regulation of the persistent infection of PCLV in Aag-2 cells.

Besides piRNA clusters, NIRVS can also be mapped to coding sequences of genes and be expressed, with a possible role in antiviral immune function (Pischedda et al., 2019). Examples of such genes are annotated as RdRp- and nucleocapsid-encoding genes of rhabdoviruses.

\section{SYSTEMIC RESPONSE TRIGGERED BY Dicer-2}

Besides its crucial function in the antiviral exo-RNAi pathway Dicer-2 was also proposed to have a role as a PAMP-recognition receptor $(\mathrm{PRR})$ during viral infections. The $\mathrm{DExD} / \mathrm{H}$ helicase domains of Dcr-2 enzymes in insects and the RIG-I-like receptors (RLRs), that trigger interferon I responses in mammals, were shown to be phylogenetically related and could be grouped together in the family of duplex RNA-activated ATPases (DRAs) family (Luo et al., 2013). A recent study mainly focusing on Drosophila suggests that Dcr-2 may preferentially interact with DVGs in the cytoplasm and promote the interaction with retrotransposons to synthesize vDNA (Poirier et al., 2018). Evidence also exists that vDNA could act as a systemic signal in mosquitoes and prime the immune response against infection of homologous virus (Goic et al., 2016; Poirier et al., 2018).

In addition, an involvement of the helicase domain of Dcr-2 was shown in the production of Vago, a secreted peptide with antiviral function characterized by a single von Willebrand $\mathrm{C}$ domain. It was observed that infection of WNV in a cell line of Cx. quinquefasciatus (Hsu) induced the expression of the secreted peptide CxVago in a Dcr-2-dependent manner (Paradkar et al., 2012). The induction of CxVago expression was also observed after injection of Cx. pipiens f. molestus mosquitoes with Kunjin virus (KUNV; Flaviviridae) and after infection of the Ae. albopictus RML12 cell line with DENV (Paradkar et al., 2012, 2014), but not after injection of Ae. aegypti mosquitoes with DENV (Asad et al., 2018).

In the Hsu cell line, it was demonstrated that CxVago exhibited antiviral effects through the induction of the JAK/STAT pathway (Paradkar et al., 2012). In order to induce the STAT-dependent target gene vir-1 and to inhibit WNV replication, CxVago signaling required CxJAK (Hopscotch homolog) but not the classical CxDome receptor for the unpaired ligands, leading to the speculation of the antiviral response occurring through an alternative JAK receptor (Paradkar et al., 2012). Another study employing the Hsu cell line documented that activation of CxVago production following WNV infection required the TRAF adaptor protein and the Rel $2 \mathrm{NF}-\kappa \mathrm{B}$ transcription factor and confirmed the involvement of Dcr- 2 in the activation mechanism (Paradkar et al., 2014). In another cell line, Aag-2, the antiviral effect of Wolbachia infection was shown to be mediated by up-regulation of AeVago1 (Asad et al., 2018).

The mechanistic details of the induction of CxVago by arbovirus infection and its activation of the JAK/STAT signaling pathway and antiviral effects have only been demonstrated in the Hsu cell line and not in mosquitoes. Similarly, while AeVago1 is 
induced during Wolbachia infection in Ae. aegypti mosquitoes, its functional involvement in repression of DENV infection remains to be demonstrated in vivo.

Other mechanisms of spread of an antiviral signal have been proposed, for instance in the $\mathrm{U} 4.4$ cell line, that requires direct contact between adjacent cells. The systemic signal, that is proposed to move from cell to cell through gap junctions or cytoplasmic bridges, may consist of viral dsRNAs/siRNAs. During infections with recombinant SFV expressing tombovirus p19 (which sequesters siRNA but not dsRNA) spread of infection was stimulated rather than viral replication in initially infected cells (Attarzadeh-Yazdi et al., 2009).

\section{THE FUNCTION OF THE MIRNA PATHWAY DURING ARBOVIRUS INFECTION}

Comparison of the rates of evolution among Drosophila species showed the accelerated evolution of genes in the exo-RNAi pathway with respect to miRNA genes, which was contributed to the molecular arms race between host antiviral response and pathogenic virus infection (Obbard et al., 2006, 2011). By contrast, another study demonstrated that genes in both miRNA and exo-RNAi pathways underwent rapid diversifying selection among different populations of Ae. aegypti (Bernhardt et al., 2012). While the causes of such accelerated evolution of miRNA genes remain to be established and may not be related to arbovirus infections, it is nevertheless of interest to consider studies that investigated the involvement of miRNAs during arbovirus infections (reviews by Asgari, 2014; Hussain et al., 2016; Monsanto-Hearne and Johnson, 2018). Intriguingly, a significant positive correlation was found between the midgut escape barrier for DENV infection and nucleotide diversity indices in $d c r-2$ but also in $d c r-1$ (Bernhardt et al., 2012).

Many studies concerning the interaction of arbovirus infection with the miRNA pathway are descriptive and involve the cataloging of miRNAs that show differential expression following arbovirus infection in mosquitoes (mostly whole body but also midgut and saliva; Zhou et al., 2014; Maharaj et al., 2015; Liu et al., 2016) or mosquito-derived cell lines, which is followed by the prediction of cellular target genes by in silico analysis or by correlation of expression with host transcripts (review by Monsanto-Hearne and Johnson, 2018). A complex response is often recorded in which particular patterns can be observed during the course of infection with different arboviruses (DENV, CHIKV, ZIKV) (Saldaña et al., 2017; review by Monsanto-Hearne and Johnson, 2018) but for which the functional relevance in vivo remains untested. Gene ontology (GO) and Kyoto Encyclopedia of Genes and Genomes (KEGG) analysis reveals the identification of immune genes among different predicted cellular targets (Liu et al., 2015, 2016; Xing et al., 2016). However, in some studies, no significant changes in miRNA abundance are observed following arbovirus infection (Miesen et al., 2016b; Ferreira et al., 2018).

Functional studies of the interaction of miRNAs with arbovirus infection were carried out in Aag-2 cells that are amenable to RNAi-mediated gene silencing and can be easily transfected with reporter/sensor/expression constructs. Such studies established a miRNA-mediated mechanism by which Wolbachia bacterial endosymbionts may inhibit DENV infection in Ae. aegypti mosquitoes, i.e., through the induction of aae-miR-2940 and inhibition of its target Dnmt2 (encoding a DNA methyltransferase) (Zhang et al., 2013). In another study, infection of mosquito cell lines by the Kunjin strain of WNV becomes restricted following down-regulation of aaemiR-2940 and its target gene encoding the metalloprotease m41 FtsH (which is positively regulated by the miRNA) (Slonchak et al., 2014). Also aae-miR-375 enhances DENV infection in Aag-2 cells, an observation which can be correlated with increased expression of cactus, which encodes an inhibitor of the immune regulator REL1, an NF- $\kappa$ B transcription factor (Hussain et al., 2013). Regarding the mosquito-borne alphavirus North American eastern equine encephalitis virus (EEEV), it was reported that the integrity of a region in the $3^{\prime} \mathrm{UTR}$, which coincides with binding sites for miR-142-3p in mammalian cells, was important for viral replication in mosquito C6/36 cells and infection of Ochlerotatus taeniorhynchus vector mosquitoes (Trobaugh et al., 2014).

Functional studies in Ae. albopictus mosquitoes through the injection of miRNA mimics or antagomirs revealed a positive role for the midgut-specific aae-miR-281 to regulate DENV replication (Zhou et al., 2014). Sensor constructs that harbor aae-miR-281 target sites from the $5^{\prime}$-UTR of DENV were positively affected following administration of aae-miR-281 in C6/36 cells, indicating a direct interaction between miR-281 and the DENV genome (Zhou et al., 2014). Finally, all functional studies employing flavivirus should take into account that sfRNA could also act as an inhibitor of the miRNA pathway, in addition to the exo-RNAi pathway, as demonstrated for WNV-sfRNA in Drosophila S2 cells (Schnettler et al., 2012) (see also section "SfRNA" for discussion of the possible function of sfRNA as VSR).

miRNAs can also be produced by viruses although it is considered rather unlikely that RNA viruses produce miRNAs (discussion by Umbach and Cullen, 2009). During infection of Aag-2 and C6/36 cells with the Kunjin strain of WNV, a viral miRNA (KUN-miR-1) is produced that is derived from the $3^{\prime}$ stem-loop located at the very end of the $3^{\prime}$-UTR or sfRNA (Hussain et al., 2012). KUN-miR-1 was proposed to stimulate Kunjin virus infection through positive regulation of the target gene GATA-4, which is a transcriptional regulator of genes involved in lipid metabolism. Another example is DENVvsRNA-5 that corresponds to the first stem-loop structure at the beginning of the $3^{\prime}$-UTR of sfRNA of DENV (Hussain and Asgari, 2014). DENV-vsRNA-5 is proposed to have an autoregulatory function as it targets the ORF of DENV NS1. However, the latter work was criticized since DENV-vsRNA-5 was expressed at too low levels to be able to act as a stoichiometric inhibitor like other miRNAs (Skalsky et al., 2014). Another study has predicted several potential viral miRNAs from DENV in silico (Ospina-Bedoya et al., 2014) but they could not be experimentally verified (Miesen et al., 2016a). Production of miRNAs by arboviruses remains therefore a contentious issue and so far functional data were obtained in cell lines but not in mosquitoes. 


\section{RNAi AND THE ANTIVIRAL DEFENSE AGAINST MOSQUITO-SPECIFIC VIRUSES}

With the advent of deep sequencing techniques and an increasing interest in the mosquito microbiome, a considerable number of new viruses were identified that are related to known arboviruses belonging to the genera Flavivirus and Alphavirus, the family Rhabdoviridae and the order Bunyavirales (Blitvich and Firth, 2015; Bolling et al., 2015; Longdon et al., 2015; Marklewitz et al., 2015; Halbach et al., 2017). In general, it was observed that the taxonomic groups that encompass known arboviruses contain additional viruses that can be considered either as mosquito-specific viruses (i.e., restricted to only mosquitoes and no transmission to vertebrates) or as having "no known vector" (i.e., likely restricted to only vertebrates). In addition, many new viruses that infect mosquitoes were isolated that have a taxonomy not related to any current known arboviruses (Ma et al., 2011; Zirkel et al., 2013; Vasilakis et al., 2013; Parry and Asgari, 2018).

Because such mosquito-specific viruses do not require a period of persistence to acquire competence for transmission to a vertebrate host, they can be expected to cause pathogenic infections in mosquitoes. However, in practice, no such pathogenic infections were manifest in most instances since it was found that vertical (transovarial) transmission was the most frequent pathway for viral dispersion in the mosquito population (Blitvich and Firth, 2015; Longdon et al., 2015; Halbach et al., 2017; Parry and Asgari, 2018). Thus, mosquito-specific viruses may not differ much from arboviruses since a long-term state of equilibrium between virus and host is desirable in both cases, although differences in tissue tropism (salivary glands versus gonads) may be apparent. In such case, the same principles with respect to avoidance of clearance by the RNAi mechanism or recognition by the immune response will be found during both arbovirus and persistent mosquito-specific virus infections.

Nevertheless, it was found that some mosquito-specific viruses can encode inhibitors of RNAi, indicating their potential to spread by horizontal transmission and to cause pathogenic infections [e.g., mosinovirus (MoNV, Nodaviridae; Schuster et al., 2014] and Culex Y virus (CYV, Birnaviridae; van Cleef et al., 2014; Franzke et al., 2018). For other viruses, the presence of a VSR gene could be inferred after analysis of the profile of viral small RNAs (Aguiar et al., 2015).

During comparisons between infections of DENV and the mosquito-specific flavivirus CFAV in the mosquito cell lines C6/36 and Aag-2, much higher levels of viral small RNAs were detected for CFAV which reflected its higher replication levels (Scott et al., 2010). As is the case for DENV infections, both vsiRNAs and vpiRNAs were observed in CFAV infections of which the latter showed a ping-pong signature (A10 but not U1) in Dcr-2-defective C6/36 cells. During CFAV infections much more prominent hot spots of viral small RNA production were observed as during DENV infections.

In Anopheles mosquitoes, production of vsiRNAs is the predominant pathway for some infections of mosquito-specific flaviviruses, while in other infections viral small RNAs of many different sizes are produced corresponding to the positive strand and with a minor peak at $21 \mathrm{nt}$ (Colmant et al., 2017). During infections of Ae. aegypti cells with the negative-strand Aedes anphevirus (AeAV; unclassified in Mononegavirales order), the production of vsiRNAs is dwarfed by the presence of vpiRNAs that show a clear ping-pong pattern (A10 genome, U1 antigenome) (Parry and Asgari, 2018). As also observed in arboviruses with negative-strand genomes (Léger et al., 2013), hot spots for abundant vpiRNA production can be observed.

In Culex-derived cell lines, different patterns of viral small RNA production are observed following infection with the mosquito-specific flavivirus Calbertado virus (CLBOV; only vsiRNAs) and the mosquito-specific rhabdovirus Meridavirus (MERDV; both vsiRNAs and vpiRNAs, the latter with positive strand-bias and ping-pong signature) (Rückert et al., 2019). These differences in viral small RNAs are considered to be caused by infections with viruses of different families (Flaviviridae and Rhabdoviridae) while it is expected that infections with arboviruses and mosquito-specific viruses of the same family will generate similar patterns of viral small RNAs in similar hosts. Mosquito-specific (CLBOV) and arboviral (WNV) flaviviral infection of Culex tarsalis-derived CT cells indeed results in the generation of very comparable profiles of viral small RNAs (Rückert et al., 2019).

The high similarity between infections of mosquitospecific viruses and arboviruses that belong to the same virus family is also observed with respect to their sensitivity to Wolbachia co-infection. While both mosquitospecific and arboviral flavivirus (with (+) ssRNA genome) infections are effectively cleared by Wolbachia, bunyaviruses [with segmented (-) or ambisense ssRNA genome] are resistant to the endosymbiont and the resistant phenotype applies to both mosquito-specific and arboviral species (Schnettler et al., 2016).

In contrast to the significant production of both vsiRNAs and candidate piRNAs (the latter albeit without ping-pong signature), it was reported that miRNA expression was very little affected during infection of Ae. aegypti with the mosquito-specific flavivirus Palm Creek virus (PCV) (Lee et al., 2017). Furthermore, inhibition of the few miRNAs that showed differential expression did not result in any effects on PCV replication. As is the case for arboviruses, this study suggests at best a minor role for miRNAs in the regulation of mosquito-specific virus infection (see also section "The Function of the miRNA Pathway During Arbovirus Infection").

The above observations of viral small RNA accumulation indicate that mosquito-specific viruses can activate both the exoRNAi (siRNA) and piRNA mechanism while other unknown RNA degradation pathways are also revealed. In conclusion, there is no evidence for significant differences between infections of arboviruses and mosquito-specific viruses belonging to the same virus family with respect to the RNAi response and other resistance mechanisms, although higher amounts of viral small RNAs may accumulate in infections with mosquito-specific 
viruses because of their higher replication level, potentially leading to pathogenicity.

\section{ENGINEERING THE RdRP ENGINES OF ARBOVIRUS REPLICATION}

The ability of (arbo)viruses to induce RNAi effects and achieve VIGS is mainly determined by two properties, i.e., the encoding of VSR genes and the characteristics of the RdRp enzymes with respect to kinetics and replication capacity (O'Neal et al., 2014). Both properties should be coordinated such that an optimal balance is achieved between viral replication and avoidance of the immune response to promote viral persistence (Randall and Griffin, 2017). While the importance of VSRs to regulate persistence and pathogenicity was already mentioned (section "Evidence for Presence of VSR Genes in Arboviral Genomes"), this part will focus on the effect of the properties of the RdRP enzyme on RNA virus infections.

RdRP enzymes of RNA viruses have high error rates which result in mutation frequencies of $10^{-4}$ mutations per nucleotide copied (Sanjuan et al., 2010). Error rates are influenced by environmental factors and can be stimulated by nucleoside analogs (te Velthuis, 2014). Few data exist with respect to the characteristics of RdRP enzymes of arboviruses but recently several studies were published that investigate the effects of lower or higher fidelity RdRp variants on infection properties of alphaviruses such as SINV and CHIKV (Coffey et al., 2011; Rozen-Gagnon et al., 2014; Stapleford et al., 2015; Poirier et al., 2016).

Research focused on the role of a residue in the palm domain of RdRp of alphaviruses that regulates its replication fidelity (Rozen-Gagnon et al., 2014; Stapleford et al., 2015). When tested in Ae. aegypti mosquitoes, an antimutator variant (exhibiting higher fidelity) of CHIKV exhibited lower infection and dissemination titers which was attributed to reduced genetic diversity (Coffey et al., 2011). On the other hand, mutator variants (with lower fidelity) of CHIKV and SINV displayed replication defects in mosquito cells and reverted to wild-type or other replication competent variants (RozenGagnon et al., 2014). During infection of Drosophila S2 cells with low fidelity SINV, by contrast, no replication defect or reversion to wild-type was observed although mutator variants presented significantly lower titers than wild-type. These data illustrate the potential for engineering RdRp enzymes to affect infectivity and replication and also indicate the existence of cell-specific effects.

The (negative) effects of both antimutator and mutator variants were initially thought to be mainly caused by the reduction and augmentation of genetic diversity leading to the proposal that RdRp enzymes of RNA viruses have become optimized during evolution to be neither too accurate nor too erroneous. However, in mammalian cells it was also demonstrated that mutator variants of SINV show an increased recombination rate leading to defective interfering (DI) particle production (Poirier et al., 2016). Whether this phenomenon also occurs in mosquito cells and how it can impact the RNAi response and the establishment of persistent infections remains to be investigated.

\section{CONCLUSION}

Arbovirus infections of mosquitoes are characterized by the absence of pathogenic effects that allow their spread within the mosquito body to the salivary glands from where infection of new vertebrate hosts can be initiated. Despite the persistent character of the infections, functional viral small RNAs were produced by recombinant viruses that could confer resistance to secondary infections by unrelated arboviruses (Gaines et al., 1996; Olson et al., 1996, 2002; Powers et al., 1996; Adelman et al., 2001). However, it was not determined whether recombinant arboviruses could induce silencing of cellular genes in mosquitoes or mosquito cell lines.

Because of the persistent character of their infections, engineered arboviruses or related mosquito-specific viruses could be developed as effective gene silencing vectors in mosquitoes. RNAi is a very powerful tool to carry out reverse genetics studies but in many cases the delivery of the dsRNA trigger is limiting (Scott et al., 2013; Whitten and Dyson, 2017). Engineered RNA viruses that have incorporated cellular gene fragments could be developed as efficient silencing vectors for analysis of gene function on a gene-by-gene basis. Moreover, if high efficiency of oral infection can be achieved, libraries of viral silencing vectors can be applied in large-scale screening experiments for interrogation of gene function in physiological and developmental processes. In such applications, however, it is extremely important to avoid non-specific effects caused by damage of viral replication and activation of innate immunity, which is the reason why persistent infections of arboviruses can serve as an excellent model for the development of viral silencing vectors. In a second type of application, viral silencing vectors that target essential genes of the host can be employed as novel types of insecticides since incorporation of fragments of essential host genes in the viral genomes is predicted to increase the virulence and to induce lethality (Taning et al., 2018). Because of the specificity of hybridization of the vsiRNAs to the targeted mRNAs, knock-down of essential genes and associated lethal effects will be observed in only single or closely related species, which is an increased safety feature. However, the application of engineered viruses as specific insecticides needs careful evaluation with respect to interaction with non-target species, the stability of the genetic material and possible recombination with co-infecting natural viruses (Kolliopoulou et al., 2017).

Understanding of the parameters that determine the persistent character of arbovirus infections may have important implications for the design of silencing vectors in insects in general. In this review, an extensive discussion is presented regarding the interaction of arbovirus infections with the mosquito RNAi machinery that can serve as a background for the improvement of the capacity of arbovirus vectors to induce specific gene silencing. As already mentioned in other studies (O’Neal et al., 2014; Kolliopoulou et al., 2017), 
two important factors have emerged that determine the capacity of viruses to function as gene silencing factors: (1) the presence of VSR genes in the virus genome, and (2) the replication capacity of the virus mainly determined by the properties of the RdRp.

It remains under debate whether arboviruses encode canonical VSR genes. However, other strategies for evading the RNAi response can exist such as the shielding of viral replication complexes in membrane structures (Den Boon and Ahlquist, 2010) or the activation of other immune pathways such as JAKSTAT and Imd (Waldock et al., 2012). On the other hand, when arbovirus genomes are engineered to contain a wellcharacterized VSR gene from an unrelated RNA virus, infections become much more virulent due to higher viral replication (Myles et al., 2008). These experiments indicate that it is possible to modulate the RNAi response in arbovirus vectors through the artificial introduction of VSR genes of different strengths (O'Neal et al., 2014). It remains to be determined whether the gene silencing activity of arboviruses can be improved by the incorporation of VSR genes. An optimal level of inhibition of RNAi may exist that achieves more efficient silencing than that observed for wild-type arboviruses because of the higher levels of viral replication that are achieved. However, the occurrence of cellular damage and the activation of innate immune pathways need to be avoided because they will confuse the interpretation of the phenotypes caused by specific gene silencing.

Besides activation of the exo-RNAi pathway, which is thought to carry out the gene silencing effects, arbovirus infections in mosquitoes also can result in the abundant production of vpiRNAs. Since their involvement in antiviral defense was not clearly demonstrated (Varjak et al., 2018b), the function of vpiRNAs during viral infection remains to be elucidated. Their production may be a secondary effect of the expansion of PIWI genes in aedine and culicine mosquitoes since canonical vpiRNAs (with ping-pong signature) do not appear during arbovirus infections of anopheline mosquitoes, sandflies or midges that (presumably) have a more standard set of PIWI genes (Myles et al., 2009; Schnettler et al., 2013b; Ferreira et al., 2018; Dietrich et al., 2017b). The production of vpiRNAs in aedine and culicine mosquitoes occurs with specific patterns according to the type of virus (alphavirus, flavivirus, or bunyavirus) (Varjak et al., $2018 \mathrm{~b})$. While studies of the function of piRNAs have mainly focused on their role in transposon silencing in the germline, the production of piRNAs in somatic tissues of many insects was revealed recently (Lewis et al., 2018). piRNAs in somatic tissues do not only correspond to transposable elements but also map to protein-coding genes that suggest unknown functions in the regulation of gene expression. Open questions include whether canonical vpiRNAs are also produced during viral infections in insects that do not have an expanded PIWI gene set and whether they may function in the regulation of viral and cellular gene expression. The investigation of the functions of vpiRNAs, however, may be aggravated by their overlap in sequence with vsiRNAs. A major argument against the antiviral function of the piRNA pathway is that VSRs targeting this pathway were never identified.
An interesting observation is the production of viral DNA forms during infection which is thought to be related to antiviral defense (Nag et al., 2016; Poirier et al., 2018). This phenomenon was reported in Drosophila and mosquitoes and may be related to the abundance of retrotransposons encoding active RT enzymes in the insect genomes (Palatini et al., 2017). Viral DNA forms may be intermediates in the process of integration of viral sequences in insect genomes where they may constitute some type of immune memory against viral infections, possibly through the production of piRNAs (Olson and Bonizzoni, 2017). Integrated viral sequences that were found generally do not represent current infections and evidence of an antiviral function of EVEs and NIRVs to modulate recent arboviral infections is lacking (Varjak et al., 2018b).

Besides its role in the exo-RNAi pathway, Dcr-2 was also found to have a function in the systemic antiviral response. The helicase domain of Dcr-2 functions as a dsRNA sensor and initiates a signaling cascade for the production of secreted antiviral signaling peptides (Paradkar et al., 2012, 2014), similar to cytosolic dsRNA sensors in mammals (Luo et al., 2013). While this review focused on the antiviral RNAi pathway, abundant evidence exists regarding the existence of several other antiviral pathways in mosquitoes (e.g., Xi et al., 2008; Souza-Neto et al., 2009; Rodriguez-Andres et al., 2012; Blair and Olson, 2014; Cheng et al., 2016; Clem, 2016) that are beyond the scope of this review. Of relevance is the observation that in insects, in contrast to vertebrates, very few PRRs were identified that directly interact with PAMPs produced during viral infections (e.g., Deddouche et al., 2008; Nakamoto et al., 2012). Instead, general damage that occurs during excessive viral replication and virion production may induce a stress response that will curtail the spread of viral infections (Moreno-García et al., 2014). From this viewpoint, control of the activity of RdRp enzymes of RNA viruses is essential for evasion of innate immunity pathways. RdRp enzymes of alphaviruses that differ from wild-type with respect to fidelity were described (Rozen-Gagnon et al., 2014) but how these and other types of mutants may interact differentially with the RNAi machinery and other innate immune pathways need to be investigated in more detail.

While arboviruses establish persistent infections and trigger an RNAi response in mosquitoes, their development as silencing vectors may not be practical because of their capacity to cause disease in humans and livestock. Fortunately, mosquitospecific viruses related to arboviruses were described for which reverse genetics systems are available (Nasar et al., 2015; Junglen et al., 2017). In addition, the technique of circular polymerase extension cloning allows the straightforward assembly of reverse genetics systems for RNA viruses without the need for molecular cloning using bacterial strains (Quan and Tian, 2009; Piyasena et al., 2017). Since mosquitospecific viruses generally also cause persistent infections in mosquitoes that resemble persistent infections by arboviruses (albeit with different tissue tropism), they represent a more safe alternative to arboviruses. However, their replication properties, interaction with innate immunity and RNAi machinery, and host range need to be investigated to a much greater extent. 


\section{AUTHOR CONTRIBUTIONS}

JL and LS conceived the idea, and designed and wrote the manuscript. AK designed the figure and contributed to the revision of the text. GS revised and contributed to improve the final version of the manuscript. All authors read and approved the final version of the manuscript.

\section{ACKNOWLEDGMENTS}

LS and AK acknowledge support of this work by the project, "Target Identification and Development of Novel Approaches for Health and Environmental Applications" (MIS 5002514) which

\section{REFERENCES}

Adelman, Z. N., Anderson, M. A. E., Wiley, M. R., Murreddu, M. G., Samuel, G. H., Morazzani, E. M., et al. (2013). Cooler temperatures destabilize RNA interference and increase susceptibility of disease vector mosquitoes to viral infection. PLoS Negl. Trop. Dis. 7:e2239. doi: 10.1371/journal.pntd.0002239

Adelman, Z. N., Blair, C. D., Carlson, J. O., Beaty, B. J., and Olson, K. E. (2001). Sindbis virus-induced silencing of dengue viruses in mosquitoes. Insect Mol. Biol. 10, 265-273. doi: 10.1046/j.1365-2583.2001.00267.x

Adelman, Z. N., Sanchez-Vargas, I., Travanty, E. A., Carlson, J. O., Beaty, B. J., Blair, C. D., et al. (2002). RNA silencing of dengue virus type 2 replication in transformed C6/36 mosquito cells transcribing an inverted-repeat RNA derived from the virus genome. J. Virol. 76, 12925-12933. doi: 10.1128/jvi.76.24.1292512933.2002

Aguiar, E. R., Olmo, R. P., Paro, S., Ferreira, F. V., de Faria, I. J., Todjro, Y. M. H., et al. (2015). Sequence-independent characterization of viruses based on the pattern of viral small RNAs produced by the host. Nucleic Acids Res. 43, 6191-6206. doi: 10.1093/nar/gkv587

Akbari, O. S., Antoshechkin, I., Amrhein, H., Williams, B., Diloreto, R., Sandler, J., et al. (2013). The developmental transcriptome of the mosquito Aedes aegypti, an invasive species and major Arbovirus vector. G3 3, 1493-1509. doi: 10.1534/ g3.113.006742

Arensburger, P., Hice, R. H., Wright, J. A., Craig, N. L., and Atkinson, P. W. (2011). The mosquito Aedes aegypti has a large genome size and high transposable element load but contains a low proportion of transposon-specific piRNAs. BMC Genomics 12:606. doi: 10.1186/1471-2164-12-606

Asad, S., Parry, R., and Asgari, S. (2018). Upregulation of Aedes aegypti Vagol by Wolbachia and its effect on dengue virus replication. Insect Biochem. Mol. Biol. 92, 45-52. doi: 10.1016/j.ibmb.2017.11.008

Asgari, S. (2014). Role of microRNAs in Arbovirus/vector interactions. Viruses 6, 3514-3534. doi: 10.3390/v6093514

Atkins, G. J. (2013). The pathogenesis of alphaviruses. ISRN Virol. 2013:861912.

Attarzadeh-Yazdi, G., Fragkoudis, R., Chi, Y., Siu, R. W. C., Ülper, L., Barry, G., et al. (2009). Cell-to-cell spread of the RNA interference response suppresses Semliki forest virus (SFV) infection of mosquito cell cultures and cannot be antagonized by SFV. J. Virol. 83, 5735-5748. doi: 10.1128/JVI.02440-08

Ballinger, M. J., Bruenn, J. A., Hay, J., Czechowski, D., and Taylor, D. J. (2014). Discovery and evolution of bunyavirids in arctic phantom midges and ancient bunyavirid-like sequences in insect genomes. J. Virol. 88, 8783-8794. doi: 10. 1128/JVI.00531-14

Barletta, A. B., Silva, M. C., and Sorgine, M. H. (2012). Validation of Aedes aegypti Aag-2 cells as a model for insect immune studies. Parasit. Vectors 5:148. doi: 10.1186/1756-3305-5-148

Beaty, B. J., Rayms-Keller, A., Borucki, M. K., and Blair, C. D. (2000). LaCrosse encephalitis virus and mosquitoes: a remarkable relationship. ASM News 66, 349-357.

Bellés, X. (2010). Beyond Drosophila: RNAi in vivo and functional genomic in insects. Annu. Rev. Entomol. 55, 11-128. is implemented under the Action for the Strategic Development on the Research and Technological Sectors, funded by the Operational Programme, "Competitiveness, Entrepreneurship and Innovation" (NSRF 2014-2020) and co-financed by the Greece and the European Union (European Regional Development Fund). LS and GS also acknowledge support by the COST (European Cooperation in Science and Technology) under grant agreement No. CA15223. JL acknowledges support of this work by grants from the National Natural Science Foundation of China (31501898), the Natural Science Foundation of Guangdong Province (2017A030313152), the Pearl River S\&T Nova Program of Guangzhou (201710010094), and the Guangzhou University's 2017 training program for young top-notch personnel (BJ201712).

Bernhardt, S. A., Simmons, M. P., Olson, K. E., Beaty, B. J., Blair, C. D., and Black, W. C. (2012). Rapid intraspecific evolution of miRNA and siRNA genes in the mosquito Aedes aegypti. PLoS One 7:e44198. doi: 10.1371/journal.pone. 0044198

Black, W. C. IV, and Moore, C. G. (2005). "Population biology as a tool to study vector-borne diseases," in Biology of Disease Vectors, 2nd Edn, ed. W. C. Marquardt (Burlington, MA: Elsevier Academic), 187-206.

Blair, C. D. (2011). Mosquito RNAi is the major innate immune pathway controlling Arbovirus infection and transmission. Future Microbiol. 6, 265-277. doi: $10.2217 / \mathrm{fmb} .11 .11$

Blair, C. D., and Olson, K. E. (2014). Mosquito immune responses to Arbovirus infections. Curr. Opin. Insect Sci. 3, 22-29. doi: 10.1016/j.cois.2014.07.005

Blair, C. D., and Olson, K. E. (2015a). Arbovirus-mosquito interactions: RNAi pathway. Curr. Opin. Virol. 15, 119-126. doi: 10.1016/j.coviro.2015.10.001

Blair, C. D., and Olson, K. E. (2015b). The role of RNA interference (RNAi) in Arbovirus-vector interactions. Viruses 7, 820-843. doi: 10.3390/v7020820

Blakqori, G., Delhaye, S., Habjan, M., Blair, C. D., Sanchez-Vargas, I., Olson, K. E., et al. (2007). La Crosse bunyavirus nonstructural protein NSs serves to suppress the type I interferon system of mammalian hosts. J. Virol. 81, 4991-4999. doi: 10.1128/jvi.01933-06

Blitvich, B. J., and Firth, A. E. (2015). Insect-specific flaviviruses: a systematic review of their discovery, host range, mode of transmission, superinfection exclusion potential and genomic organization. Viruses 7, 1927-1959. doi: 10. 3390/v7041927

Bolling, B. G., Weaver, S. C., Tesh, R. B., and Vasilakis, N. (2015). Insect-specific virus discovery: significance for the Arbovirus community. Viruses 7, 49114928. doi: 10.3390/v7092851

Brackney, D. E., Beane, J. E., and Ebel, G. D. (2009). RNAi targeting of west Nile virus in mosquito midguts promotes virus diversification. PLoS Pathog. 5:e1000502. doi: 10.1371/journal.ppat.1000502

Brackney, D. E., Schirtzinger, E. A., Harrison, T. D., Ebel, G. D., and Hanley, K. A. (2015). Modulation of flavivirus population diversity by RNA interference. J. Virol. 89, 4035-4039. doi: 10.1128/JVI.02612-14

Brackney, D. E., Scott, J. C., Sagawa, F., Woodward, J. E., Miller, N. A., Schilkey, F. D., et al. (2010). C6/36 Aedes albopictus cells have a dysfunctional antiviral RNA interference response. PLoS Negl. Trop. Dis. 4:e856. doi: 10.1371/journal. pntd.0000856

Bronkhorst, A. W., and van Rij, R. P. (2014). The long and short of antiviral defense: small RNA-based immunity in insects. Curr. Opin. Virol. 7, 19-28. doi: 10.1016/j.coviro.2014.03.010

Campbell, C. L., Black, W. C. IV, Hess, A. M., and Foy, B. D. (2008). Comparative genomics of small RNA regulatory pathway components in vector mosquitoes. BMC Genomics 9:425. doi: 10.1186/1471-2164-9-425

Carissimo, G., Pondeville, E., McFarlane, M., Dietrich, I., Mitri, C., Bischoff, E., et al. (2015). Antiviral immunity of Anopheles gambiae is highly compartmentalized, with distinct roles for RNA interference and gut microbiota. Proc. Natl. Acad. Sci. U.S.A. 112, E176-E185. doi: 10.1073/pnas. 1412984112 
Chen, X.-G., Jiang, X., Gu, J., Xu, M., Wu, Y., Deng, Y., et al. (2015). Genome sequence of the Asian Tiger mosquito, Aedes albopictus, reveals insights into its biology, genetics, and evolution. Proc. Natl. Acad. Sci. U.S.A. 112, E5907E5915.

Cheng, G., Liu, Y., Wang, P., and Xiao, X. (2016). Mosquito defense strategies against viral infection. Trends Parasitol. 32, 177-186. doi: 10.1016/j.pt.2015. 09.009

Cirimotich, C. M., Scott, J. C., Phillips, A. T., Geiss, B. J., and Olson, K. E. (2009). Suppression of RNA interference increases alphavirus replication and virusassociated mortality in Aedes aegypti mosquitoes. BMC Microbiol. 9:49. doi: 10.1186/1471-2180-9-49

Clem, R. J. (2016). Arboviruses and apoptosis: the role of cell death in determining vector competence. J. Gen. Virol. 97, 1033-1036. doi: 10.1099/jgv.0.000429

Coffey, L. L., Beeharry, Y., Bordería, A. V., Blanc, H., and Vignuzzi, M. (2011). Arbovirus high fidelity variant loses fitness in mosquitoes and mice. Proc. Natl. Acad. Sci. U.S.A. 108, 16038-16043. doi: 10.1073/pnas.1111650108

Colmant, A. M. G., Hobson-Peters, J., Bielefeldt-Ohmann, H., van den Hurk, A. F., Hall-Mendelin, S., Chow, W. K., et al. (2017). A new clade of insect-specific flaviviruses from Australian Anopheles mosquitoes displays species-specific host restriction. mSphere 2:e0262-17. doi: 10.1128/mSphere.00262-17

Crochu, S., Cook, S., Attoui, H., Charrel, R. N., De Chesse, R., Belhouchet, M., et al. (2004). Sequences of flavivirus-related RNA viruses persist in DNA form integrated in the genome of Aedes spp. mosquitoes. J. Gen. Virol. 85, 1971-1980. doi: 10.1099/vir.0.79850-0

Deddouche, S., Matt, N., Budd, A., Mueller, S., Kemp, C., Galiana-Arnoux, D., et al. (2008). The DExD/H-box helicase Dicer-2 mediates the induction of antiviral activity in Drosophila. Nat. Immunol. 9, 1425-1432. doi: 10.1038/ni.1664

Den Boon, J. A., and Ahlquist, P. (2010). Organelle-like membrane compartmentalization of positive-strand RNA virus replication factories. Annu. Rev. Microbiol. 64, 241-256. doi: 10.1146/annurev.micro.112408.134012

Dietrich, I., Jansen, S., Fall, G., Lorenzen, S., Rudolf, M., Huber, K., et al. (2017a). RNA interference restricts rift valley fever virus in multiple insect systems. mSphere 2:e0090-17. doi: 10.1128/mSphere.00090-17

Dietrich, I., Shi, X., McFarlane, M., Watson, M., Blomström, A.-L., Skelton, J. K., et al. (2017b). The antiviral RNAi response in vector and non-vector cells against Orthobunyaviruses. PLoS Negl. Trop. Dis. 11:e0005272. doi: 10.1371/ journal.pntd.0005272

Donald, C. L., Kohl, A., and Schnettler, E. (2012). New insights into control of Arbovirus replication and spread by insect RNA interference pathways. Insects 3, 511-531. doi: 10.3390/insects3020511

Donald, C. L., Varjak, M., Aguiar, E. R. G. R., Marques, J. T., Sreenu, V. B., Schnettler, E., et al. (2018). Antiviral RNA interference activity in cells of the predatory mosquito, Toxorhynchites amboinensis. Viruses 10:694. doi: 10.3390/ v10120694

Dowling, D., Pauli, T., Donath, A., Meusemann, K., Podsiadlowski, L., Petersen, M., et al. (2016). Phylogenetic origin and diversification of RNAi pathway genes in insects. Genome Biol. Evol. 8, 3784-3793. doi: 10.1093/gbe/evw281

Dutuze, M. F., Nzayirambaho, M., Mores, C. N., and Christofferson, R. C. (2018). A review of bunyamwera, batai, and ngari viruses: understudied Orthobunyaviruses with potential one health implications. Front. Vet. Sci. 5:69. doi: 10.3389/fvets.2018.00069

Ebel, G. D., Rochlin, I., Longacker, J., and Kramer, L. D. (2005). Culex restuans (Diptera: Culicidae) relative abundance and vector competence for West Nile Virus. J. Med. Entomol. 42, 838-843. doi: 10.1093/jmedent/42.5.838

Ferreira, F. V., Aguiar, E. R. G. R., Olmo, R. P., de Oliveira, K. P. V., Silva, E. G., Sant'Anna, M. R. V., et al. (2018). The small non-coding RNA response to virus infection in the Leishmania vector Lutzomyia longipalpis. PLoS Negl. Trop. Dis. 12:e0006569. doi: 10.1371/journal.pntd.0006569

Fire, A., Xu, S., Montgomery, M. K., Kostas, S. A., Driver, S. E., and Mello, C. C. (1998). Potent and specific genetic interference by double-stranded RNA in Caenorhabditis elegans. Nature 391, 806-811. doi: 10.1038/35888

Fort, P., Albertini, A., Van-Hua, A., Berthomieu, A., Roche, S., Delsuc, F., et al. (2012). Fossil rhabdoviral sequences integrated into arthropod genomes: ontogeny, evolution, and potential functionality. Mol. Biol. Evol. 29, 381-390. doi: 10.1093/molbev/msr226

Fragkoudis, R., Attarzadeh-Yazdi, G., Nash, A. A., Fazakerley, J. K., and Kohl, A. (2009). Advances in dissecting mosquito innate immune responses to Arbovirus infection. J. Gen. Virol. 90, 2061-2072. doi: 10.1099/vir.0.013201-0
Fragkoudis, R., Chi, Y., Siu, R. W., Barry, G., Attarzadeh-Yazdi, G., Merits, A., et al. (2008). Semliki Forest virus strongly reduces mosquito host defence signaling. Insect Mol. Biol. 17, 647-656. doi: 10.1111/j.1365-2583.2008.00834.x

Franz, A. W. E., Kantor, A. M., Passarelli, A. L., and Clem, R. J. (2015). Tissue barriers to Arbovirus infection in mosquitoes. Viruses 7, 3741-3767. doi: 10. 3390/v7072795

Franz, A. W. E., Sanchez-Vargas, I., Adelman, Z. N., Blair, C. D., Beaty, B. J., James, A. A., et al. (2006). Engineering RNA interference-based resistance to dengue virus type 2 in genetically modified Aedes aegypti. Proc. Natl. Acad. Sci. U.S.A. 103, 4198-4203. doi: 10.1073/pnas.0600479103

Franz, W. E. A., Sanchez-Vargas, I., Raban, R. R., Black, W. C. IV, James, A. A., and Olson, K. E. (2014). Fitness impact and stability of a transgene conferring resistance to dengue-2 virus following introgression into a genetically diverse Aedes aegypti strain. PLoS Negl. Trop. Dis. 5:e2833. doi: 10.1371/journal.pntd. 0002833

Franzke, K., Leggewie, M., Sreenu, V. B., Jansen, S., Heitmann, A., Welch, S. R., et al. (2018). Detection, infection dynamics and small RNA response against Culex Y virus in mosquito-derived cells. J. Gen. Virol. 99, 1739-1745. doi: 10.1099/jgv.0.001173

Fredericks, A. C., Wallace, L. E., Russell, T. A., Davidson, A. D., Fernandez-Sesma, A., and Maringer, K. (2019). Aedes aegypti (Aag2)-derived clonal mosquito cell lines reveal the impact of pre-existing persistent infection with the insectspecific bunyavirus Phasi Charoen-like virus on Arbovirus replication. BioRxiv [Preprint]. doi: 10.1101/596205

Fros, J. J., Miesen, P., Vogels, C. B., Gaibani, P., Sambri, V., Martina, B. E., et al. (2015). Comparative Usutu and West Nile virus transmission potential by local Culex pipiens mosquitoes in north-western Europe. One Health 1, 31-36. doi: 10.1016/j.onehlt.2015.08.002

Gaines, P. J., Olson, K. E., Higgs, S., Powers, A. M., Beaty, B. J., and Blair, C. D. (1996). Pathogen-derived resistance to dengue type 2 virus in mosquito cells by expression of the premembrane coding region of the viral genome. J. Virol. 70, 2132-2137.

Gammon, D. B., and Mello, C. C. (2015). RNA interference-mediated antiviral defense in insects. Curr. Opin. Insect Sci. 8, 111-120. doi: 10.1016/j.cois.2015. 01.006

Girard, Y. A., Popov, V., Wen, J., Han, V., and Higgs, S. (2005). Ultrastructural study of West Nile virus pathogenesis in Culex pipiens quinquefasciatus (Diptera: Culicidae). J. Med. Entomol. 42, 429-444. doi: 10.1603/00222585(2005)042\%5B0429:usownv\%5D2.0.co;2

Girardi, E., Miesen, P., Pennings, B., Frangeul, L., Saleh, M.-C., and van Rij, R. P. (2017). Histone-derived piRNA biogenesis depends on the ping-pong partners Piwi5 and Ago3 in Aedes aegypti. Nucleic Acids Res. 45, 4881-4892. doi: 10.1093/nar/gkw1368

Göertz, G. P., Fros, J. J., Miesen, P., Vogels, C. B. F., van der Bent, M. L., Geertsema, C., et al. (2016). Noncoding subgenomic flavivirus RNA is processed by the mosquito RNA interference machinery and determines west Nile virus transmission by Culex pipiens mosquitoes. J. Virol. 90, 10145-10159. doi: 10. 1128/JVI.00930-16

Goic, B., Stapleford, K. A., Frangeul, L., Doucet, A. J., Gausson, V., Blanc, H., et al. (2016). Virus-derived DNA drives mosquito vector tolerance to arboviral infection. Nat. Commun. 7:12410. doi: 10.1038/ncomms 12410

Halbach, R., Junglen, S., and van Rij, R. P. (2017). Mosquito-specific and mosquitoborne viruses: evolution, infection, and host defense. Curr. Opin. Insect Sci. 22, 16-27. doi: 10.1016/j.cois.2017.05.004

Handler, D., Meixner, K., Pizka, M., Lauss, K., Schmied, C., Gruber, F. S., et al. (2013). The genetic makeup of the Drosophila piRNA pathway. Mol. Cell 50, 762-777. doi: 10.1016/j.molcel.2013.04.031

Hardy, J. L., Houk, E. J., Kramer, L. D., and Reeves, W. C. (1983). Intrinsic factors affecting vector competence of mosquitoes for arboviruses. Annu. Rev. Entomol. 28, 229-262. doi: 10.1146/annurev.en.28.010183.001305

Hess, A. M., Prasad, A. N., Ptitsyn, A., Ebel, G. D., Olson, K. E., Barbacioru, C., et al. (2011). Small RNA profiling of Dengue virus-mosquito interactions implicates the PIWI RNA pathway in anti-viral defense. BMC Microbiol. 11:45. doi: 10.1186/1471-2180-11-45

Huang, Y.-J. S., Higgs, S., Horne, K. M., and Vanlandingham, D. L. (2014). Flavivirus-mosquito interactions. Viruses 6, 4703-4730. doi: 10.3390/v6114703

Hussain, M., and Asgari, S. (2014). MicroRNA-like viral small RNA from Dengue virus 2 autoregulates its replication in mosquito cells. 
Proc. Natl. Acad. Sci. U.S.A. 111, 2746-2751. doi: 10.1073/pnas.132012 3111

Hussain, M., Etebari, K., and Asgari, S. (2016). Functions of small RNAs in mosquitoes. Adv. Insect Physiol. 51, 189-222. doi: 10.1016/bs.aiip.2016.04.001

Hussain, M., Torres, S., Schnettler, E., Funk, A., Grundhoff, A., Pijlman, G. P., et al. (2012). West Nile virus encodes a micro-RNA-like small RNA in the 3' untranslated region which up-regulates GATA4 mRNA and facilitates virus replication in mosquito cells. Nucleic Acids Res. 40, 2210-2223. doi: 10.1093/ nar/gkr848

Hussain, M., Walker, T., O'Neill, S. L., and Asgari, S. (2013). Blood meal induced microRNA regulates development and immune associated genes in the Dengue mosquito vector, Aedes aegypti. Insect Biochem. Mol. Biol. 43, 146-152. doi: 10.1016/j.ibmb.2012.11.005

Huvenne, H., and Smagghe, G. (2010). Mechanisms of dsRNA uptake in insects and potential of RNAi for pest control: a review. J. Insect Physiol. 56, 227-235. doi: $10.1016 /$ j.jinsphys.2009.10.004

Ishizu, H., Iwasaki, Y. W., Hirakata, S., Ozaki, H., Iwasaki, W., Siomi, H., et al. (2015). Somatic primary piRNA biogenesis driven by cis-acting RNA elements and trans-acting Yb. Cell Rep. 12, 429-440. doi: 10.1016/j.celrep.2015.06.035

Joga, M. R., Zotti, M. J., Smagghe, G., and Christiaens, O. (2016). RNAi Efficiency, systemic properties, and novel delivery methods for pest insect control: what we know so far. Front. Physiol. 7:553. doi: 10.3389/fphys.2016.00553

Junglen, S., Korries, M., Grasse, W., Wieseler, J., Kopp, A., Hermanns, K., et al. (2017). Host range restriction of insect-specific Flaviviruses occurs at several levels of the viral life cycle. mSphere 2:e00375-16. doi: 10.1128/mSphere. 00375-16

Kakumani, P. K., Ponia, S. S., S, R. K., Sood, V., Chinnappan, M., Banerjea, A. C., et al. (2013). Role of RNA interference (RNAi) in dengue virus replication and identification of NS4B as an RNAi suppressor. J. Virol. 87, 8870-8883. doi: 10.1128/JVI.02774-12

Katzourakis, A., and Gifford, R. J. (2010). Endogenous viral elements in animal genomes. PLoS Genet. 6:e1001191. doi: 10.1371/journal.pgen.1001191

Keene, K. M., Foy, B. D., Sanchez-Vargas, I., Beaty, B. J., Blair, C. D., and Olson, K. E. (2004). RNA interference acts as a natural antiviral response to O'nyongnyong virus (Alphavirus; Togaviridae) infection of Anopheles gambiae. Proc. Natl. Acad. Sci. U.S.A. 101, 17240-17245. doi: 10.1073/pnas.0406983101

Khoo, C. C. H., Doty, J. B., Heersink, M. S., Olson, K. E., and Franz, A. W. E. (2013). Transgene-mediated suppression of the RNA interference pathway in Aedes aegypti interferes with gene silencing and enhances Sindbis virus and dengue virus type 2 replication. Insect Mol. Biol. 22, 104-114. doi: 10.1111/imb.12008

Khoo, C. C. H., Piper, J., Sanchez-Vargas, I., Olson, K. E., and Franz, A. W. E. (2010). The RNA interference pathway affects midgut infection- and escape barriers for Sindbis virus in Aedes aegypti. BMC Microbiol. 10:130. doi: 10.1186/ 1471-2180-10-130

Kingsolver, M. B., Huang, Z., and Hardy, R. W. (2013). Insect antiviral innate immunity: pathways, effectors, and connections. J. Mol. Biol. 425, 4921-4936. doi: 10.1016/j.jmb.2013.10.006

Kolliopoulou, A., Taning, C. N. T., Smagghe, G., and Swevers, L. (2017). Viral delivery of dsRNA for control of insect agricultural pests and vectors of human disease: prospects and challenges. Front. Physiol. 8:399. doi: 10.3389/fphys.2017. 00399

Lambrechts, L., and Scott, T. W. (2009). Mode of transmission and the evolution of Arbovirus virulence in mosquito vectors. Proc. Biol. Sci. 276, 1369-1378. doi: 10.1098/rspb.2008.1709

Lee, M., Etebari, K., Hall-Mendelin, S., van den Hurk, A. F., Hobson-Peters, J., Vatipally, S., et al. (2017). Understanding the role of microRNAs in the interaction of Aedes aegypti mosquitoes with an insect-specific flavivirus. J. Gen. Virol. 98, 1892-1903. doi: 10.1099/jgv.0.000832

Léger, P., Lara, E., Jagla, B., Sismeiro, O., Mansuroglu, Z., Coppée, Y., et al. (2013). Dicer-2- and Piwi-mediated RNA interference in rift valley fever virus-infected mosquito cells. J. Virol. 87, 1631-1648. doi: 10.1128/JVI.02795-12

Lewis, S. H., Quarles, K. A., Yang, Y., Tanguy, M., Frézal, L., Smith, S. A., et al. (2018). Pan-arthropod analysis reveals somatic piRNAs as an ancestral defence against transposable elements. Nat. Ecol. Evol. 2, 174-181. doi: 10.1038/s41559017-0403-4

Lewis, S. H., Salmela, H., and Obbard, D. J. (2016). Duplication and diversification of dipteran argonaute genes, and the evolutionary divergence of Piwi and aubergine. Genome Biol. Evol. 8, 507-518. doi: 10.1093/gbe/evw018
Linsen, S. E. V., de Wit, E., Janssens, G., Heater, S., Chapman, L., Parkin, R. K., et al. (2009). Limitations and possibilities of small RNA digital gene expression profiling. Nat. Meth. 6, 474-476. doi: 10.1038/nmeth0709-474

Linthicum, K. J., Britch, S. C., and Anyamba, A. (2016). Rift valley fever: an emerging mosquito-borne disease. Annu. Rev. Entomol. 61, 395-415. doi: 10. 1146/annurev-ento-010715-023819

Liu, H., Fu, Y., Jiang, D., Li, G., Xie, J., Cheng, J., et al. (2010). Widespread horizontal gene transfer from double-stranded RNA viruses to eukaryotic nuclear genomes. J. Virol. 84, 11876-11887. doi: 10.1128/JVI.00955-10

Liu, Y., Zhou, Y., Wu, J., Zheng, P., Li, Y., Zheng, X., et al. (2015). The expression profile of Aedes albopictus miRNAs is altered by dengue virus serotype-2 infection. Cell Biosci. 5:16. doi: 10.1186/s13578-015-0009-y

Liu, Y.-X., Li, F.-X., Liu, Z.-Z., Lia, Z.-R., Zhou, Y.-H., Zhang, H., et al. (2016). Integrated analysis of miRNAs and transcriptomes in Aedes albopictus midgut reveals the differential expression profiles of immune-related genes during dengue virus serotype-2 infection. Insect Sci. 23, 377-385. doi: 10.1111/17447917.12339

Longdon, B., Murray, G. G. R., Palmer, W. J., Day, J. P., Parker, D. J., Welch, J. J., et al. (2015). The evolution, diversity, and host associations of rhabdoviruses. Virus Evol. 1:vev014.

Lopez, S. B. G., Guimarães-Ribero, V., Rodriguez, J. V. G., Dorand, F. A. P. S., Salles, T. S., Sá-Guimarães, T. E., et al. (2019). RNAi-based bioinsecticide for Aedes mosquito control. Sci. Rep. 9:4038. doi: 10.1038/s41598-019-39666-5

Luo, D., Kohlway, A., and Pyle, A. M. (2013). Duplex RNA activated ATPases (DRAs): platforms for RNA sensing, signaling and processing. RNA Biol. 10, 111-120. doi: 10.4161/rna.22706

Ma, M., Huang, Y., Gong, Z., Zhuang, L., Li, C., Yang, H., et al. (2011). Discovery of DNA viruses in wild-caught mosquitoes using small RNA high throughput sequencing. PLoS One 6:e24758. doi: 10.1371/journal.pone.0024758

Macias, V., Coleman, J., Bonizzoni, M., and James, A. A. (2014). piRNA pathway gene expression in the malaria vector mosquito Anopheles stephensi. Insect Mol. Biol. 23, 579-586. doi: 10.1111/imb.12106

Maharaj, P. D., Widen, S. G., Huang, J., Wood, T. G., and Thangamani, S. (2015). Discovery of mosquito saliva MicroRNAs during CHIKV infection. PLoS Negl. Trop. Dis. 9:e0003386. doi: 10.1371/journal.pntd.0003386

Marklewitz, M., Zirkel, F., Kurth, A., Drosten, C., and Junglen, S. (2015). Evolutionary and phenotypic analysis of live virus isolates suggests arthropod origin of a pathogenic RNA virus family. Proc. Natl. Acad. Sci. U.S.A. 112, 7536-7541. doi: 10.1073/pnas.1502036112

Mathur, G., Sanchez-Vargas, I., Alvarez, D., Olson, K. E., Marinotti, O., and James, A. A. (2010). Transgene-mediated suppression of dengue viruses in the salivary glands of the yellow fever mosquito, Aedes aegypti. Insect Mol. Biol. 19, 753-763. doi: $10.1111 / j .1365-2583.2010 .01032 . x$

Mathur, K., Anand, A., Dubey, S. K., Sanan-Mishra, N., Bhatnagar, R. K., and Sunil, S. (2016). Analysis of chikungunya virus proteins reveals that non-structural proteins nsP2 and nsP3 exhibit RNA interference (RNAi) suppressor activity. Sci. Rep. 6:38065. doi: 10.1038/srep38065

McFarlane, M., Arias-Goeta, C., Martin, E., O’Hara, Z., Lulla, A., Mousson, L., et al. (2014). Characterization of Aedes aegypti innate-immune pathways that limit chikungunya virus replication. PLoS Negl. Trop. Dis. 8:e2994. doi: 10.1371/ journal.pntd.0002994

Miesen, P., Girardi, E., and van Rij, R. P. (2015). Distinct sets of PIWI proteins produce Arbovirus and transposon-derived piRNAs in Aedes aegypti mosquito cells. Nucleic Acids Res. 43, 6545-6556. doi: 10.1093/nar/gkv590

Miesen, P., Ivens, A., Buck, A. H., and van Rij, R. P. (2016a). Small RNA profiling in dengue virus 2-infected Aedes mosquito cells reveals viral piRNAs and novel host miRNAs. PLoS Negl. Trop. Dis. 10:e0004452. doi: 10.1371/journal.pntd. 0004452

Miesen, P., Joosten, J., and van Rij, R. P. (2016b). PIWIs go viral: Arbovirus-derived piRNAs in vector mosquitoes. PLoS Pathog. 12:e1006017. doi: 10.1371/journal. ppat.1006017

Monsanto-Hearne, V., and Johnson, K. N. (2018). miRNAs in insects infected by animal and plant viruses. Viruses 10:E354. doi: 10.3390/v1007 0354

Moon, S. L., Dodd, B. J. T., Brackney, D. E., Wilusz, C. J., Ebel, G. D., and Wilusz, J. (2015). Flavivirus sfRNA suppresses antiviral RNA interference in cultured cells and mosquitoes and directly interacts with the RNAi machinery. Virology 485 , 322-329. doi: 10.1016/j.virol.2015.08.009 
Morazzani, E. M., Wiley, M. R., Murreddu, M. G., Adelman, Z. N., and Myles, K. M. (2012). Production of virus-derived ping-pong-dependent piRNA-like small RNAs in the mosquito soma. PLoS Pathog. 8:e1002470. doi: 10.1371/journal. ppat. 1002470

Moreno-García, M., Condé, R., Bello-Bedoy, R., and Lanz-Mendoza, H. (2014). The damage threshold hypothesis and the immune strategies of insects. Infect. Genet. Evol. 24, 25-33. doi: 10.1016/j.meegid.2014.02.010

Myles, K. M., Morazzani, E. M., and Adelman, Z. N. (2009). Origins of alphavirusderived small RNAs in mosquitoes. RNA Biol. 6, 387-391. doi: 10.4161/rna.6.4. 8946

Myles, K. M., Wiley, M. R., Morazzani, E. M., and Adelman, Z. N. (2008). Alphavirus-derived small RNAs modulate pathogenesis in disease vector mosquitoes. Proc. Natl. Acad. Sci. U.S.A. 105, 19938-19943. doi: 10.1073/pnas. 0803408105

Nag, D. K., Brecher, M., and Kramer, L. D. (2016). DNA forms of arboviral RNA genomes are generated following infection in mosquito cell cultures. Virology 498, 164-171. doi: 10.1016/j.virol.2016.08.022

Nakamoto, M., Moy, R. H., Xu, J., Bambina, S., Yasunaga, A., Shelly, S. S., et al. (2012). Virus recognition by Toll-7 activates antiviral autophagy in Drosophila. Immunity 36, 658-667. doi: 10.1016/j.immuni.2012.03.003

Nasar, F., Gorchakov, R. V., Tesh, R. B., and Weaver, S. C. (2015). Eilat virus host range restriction is present at multiple levels of the virus life cycle. J. Virol. 89, 1404-1418. doi: 10.1128/JVI.01856-14

Nene, V., Wortman, J. R., Lawson, D., Haas, B., Kodira, C., Tu, Z. J., et al. (2007). Genome sequence of Aedes aegypti, a major Arbovirus vector. Science 316, 1718-1723.

Obbard, D. J., Jiggins, F. M., Bradshaw, N. J., and Little, T. J. (2011). Recent and recurrent selective sweeps of the antiviral RNAi gene Argonaute-2 in three species of Drosophila. Mol. Biol. Evol. 28, 1043-1056. doi: 10.1093/molbev/ msq280

Obbard, D. J., Jiggins, F. M., Halligan, D. L., and Little, T. J. (2006). Natural selection drives extremely rapid evolution in antiviral RNAi genes. Curr. Biol. 16, 580-585. doi: 10.1016/j.cub.2006.01.065

Olson, K. E., Adelman, Z. N., Travanty, E. A., Sanchez-Vargas, I., Beaty, B. J., and Blair, C. D. (2002). Developing Arbovirus resistance in mosquitoes. Insect Biochem. Mol. Biol. 32, 1333-1343. doi: 10.1016/s0965-1748(02)00096-6

Olson, K. E., and Bonizzoni, M. (2017). Nonretroviral integrated RNA viruses in arthropod vectors: an occasional event or something more? Curr. Opin. Insect Sci. 22, 45-53. doi: 10.1016/j.cois.2017.05.010

Olson, K. E., Higgs, S., Gaines, P. J., Powers, A. M., Davis, B. S., Kamrud, K. I., et al. (1996). Genetically engineered resistance to dengue-2 virus transmission in mosquitoes. Science 272, 884-886. doi: 10.1126/science.272.5263.884

O’Neal, S. T., Samuel, G. H., Adelman, Z. N., and Myles, K. M. (2014). Mosquitoborne viruses and suppressors of invertebrate antiviral RNA silencing. Viruses 6, 4314-4331. doi: 10.3390/v6114314

Ospina-Bedoya, M., Campillo-Pedroza, N., Franco-Salazar, J. P., and GallegoGomez, J. C. (2014). Computational identification of dengue virus MicroRNAlike structures and their cellular targets. Bioinform. Biol. Insights 8, 169-176. doi: 10.4137/BBI.S13649

Palatini, U., Miesen, P., Carballar-Lejarazu, R., Ometto, L., Rizzo, E., Tu, Z., et al. (2017). Comparative genomics shows that viral integrations are abundant and express piRNAs in the arboviral vectors Aedes aegypti and Aedes albopictus. BMC Genomics 18:512. doi: 10.1186/s12864-017-3903-3

Paradkar, P. N., Duchemin, J.-B., Voysey, R., and Walker, P. J. (2014). Dicer2-dependent activation of Culex vago occurs via the TRAF-Rel2 signaling pathway. PLoS Negl. Trop. Dis. 8:e2823. doi: 10.1371/journal.pntd.0002823

Paradkar, P. N., Trinidad, L., Voysey, R., Duchemin, J. B., and Walker, P. J. (2012). Secreted Vago restricts West Nile virus infection in Culex mosquito cells by activating the Jak-STAT pathway. Proc. Natl. Acad. Sci. U.S.A. 109, 18915-18920. doi: 10.1073/pnas.1205231109

Parry, R., and Asgari, S. (2018). Aedes anphevirus (AeAV): an insect-specific virus distributed worldwide in Aedes aegypti mosquitoes that has complex interplays with Wolbachia and dengue virus infection in cells. J. Virol. 92, 1-19. doi: 10.1128/JVI.00224- 18

Pijlman, G. P. (2014). Flavivirus RNAi suppression: decoding non-coding RNA. Curr. Opin. Virol. 7, 55-60. doi: 10.1016/j.coviro.2014.04.002

Pischedda, E., Scolari, F., Valerio, F., Carballar-Lejarazú, R., Catapano, P. L., Waterhouse, R. M., et al. (2019). Insights into an unexplored component of the mosquito repeatome: distribution and variability of viral sequences integrated into the genome of the arboviral vector Aedes albopictus. Front. Genet. 10:93. doi: $10.3389 /$ fgene. 2019.00093

Pitaluga, A. N., Masin, P. W., and Traub-Cseko, Y. M. (2008). Non-specific antiviral response detected in RNA-treated cultured cells of the sandfly, Lutzomyia longipalpis. Dev. Comp. Immunol. 32, 191-197. doi: 10.1016/j.dci. 2007.06.008

Piyasena, T. B. H., Setoh, Y. X., Hobson-Peters, J., Newton, N. D., BielefeldtOhmann, H., McLean, B. J., et al. (2017). Infectious DNAs derived from insect-specific flavivirus genomes enable identification of pre- and post-entry host restrictions in vertebrate cells. Sci. Rep. 7:2940. doi: 10.1038/s41598-01703120-1

Poirier, E. Z., Goic, B., Tomé-Poderti, L., Frangeul, L., Boussier, J., Gausson, V., et al. (2018). Dicer-2-dependent generation of viral DNA from defective genomes of RNA viruses modulates antiviral immunity in insects. Cell Host Microbe 23, 353-365. doi: 10.1016/j.chom.2018.02.001

Poirier, E. Z., Mounce, B. C., Rozen-Gagnon, K., Hooikaas, P. J., Stapleford, K. A., Moratorio, G., et al. (2016). Low-fidelity polymerases of alphaviruses recombine at higher rates to overproduce defective interfering particles. J. Virol. 90, 2446-2454. doi: 10.1128/JVI.02921-15

Powers, A. M., Kamrud, K. I., Olson, K. E., Higgs, S., Carlson, J. O., and Beaty, B. J. (1996). Molecularly engineered resistance to California serogroup virus replication in mosquito cells and mosquitoes. Proc. Natl. Acad. Sci. U.S.A. 93, 4187-4191. doi: 10.1073/pnas.93.9.4187

Prasad, A. N., Brackney, D. E., and Ebel, G. D. (2013). The role of innate immunity in conditioning mosquito susceptibility to west Nile virus. Viruses 5, 3142-3170. doi: $10.3390 / v 5123142$

Quan, J., and Tian, J. (2009). Circular polymerase extension cloning of complex gene libraries and pathways. PLoS One 4:e6441. doi: 10.1371/journal.pone. 0006441

Randall, R. E., and Griffin, D. E. (2017). Within host RNA virus persistence: mechanisms and consequences. Curr. Opin. Virol. 23, 35-42. doi: 10.1016/j. coviro.2017.03.001

Rezza, G., Chen, R., and Weaver, S. C. (2017). O’nyong-nyong fever: a neglected mosquito-born viral disease. Pathog. Glob. Health 111, 271-275. doi: 10.1080/ 20477724.2017.1355431

Robine, N., Lau, N. C., Balla, S., Jin, Z., Okamura, K., Kuramochi-Miyagawa, S., et al. (2009). A broadly conserved pathway generates 3'UTR-directed primary piRNAs. Curr. Biol. 19, 2066-2076. doi: 10.1016/j.cub.2009.11.064

Roby, J. A., Pijlman, G. P., Wilusz, J., and Khromykh, A. A. (2014). Noncoding subgenomic flavivirus RNA: multiple functions in west Nile virus pathogenesis and modulation of host responses. Viruses 6, 404-427. doi: 10.3390/v6020404

Rodriguez-Andres, J., Rani, S., Varjak, M., Chase-Topping, M. E., Beck, M. H., Ferguson, M. C., et al. (2012). Phenoloxidase activity acts as a mosquito innate immune response against infection with Semliki forest virus. PLoS Pathog. 8:e1002977. doi: 10.1371/journal.ppat.1002977

Rozen-Gagnon, K., Stapleford, K. A., Mongelli, V., Blanc, H., Failloux, A.-B., Saleh, M.-C., et al. (2014). Alphavirus mutator variants present host specific defects and attenuation in mammalian and insect models. PLoS Pathog. 10:e1003877. doi: 10.1371/journal.ppat.1003877

Rückert, C., Bell-Sakyi, L., Fazakerley, J. K., and Fragkoudis, R. (2014). Antiviral responses of arthropod vectors: an update on recent advances. Virusdisease 25, 249-260. doi: 10.1007/s13337-014-0217-9

Rückert, C., Prasad, A. N., Garcia-Luna, S. M., Robison, A., Grubaugh, N. D., Weger-Lucarelli, J., et al. (2019). Small RNA responses of Culex mosquitoes and cell lines during acute and persistent virus infection. Insect Biochem. Mol. Biol. 109, 13-23. doi: 10.1016/j.ibmb.2019.04.008

Rückert, C., Weger-Lucarelli, J., Garcia-Luna, S. M., Young, M. C., Byas, A. D., Murrieta, R. A., et al. (2017). Impact of simultaneous exposure to Arboviruses on infection and transmission by Aedes aegypti mosquitoes. Nat. Commun. 8:15412. doi: 10.1038/ncomms15412

Sabin, L. R., Zheng, Q., Thekkat, P., Yang, J., Hannon, G. J., Gregory, B. D., et al. (2013). Dicer-2 processes diverse viral RNA species. PLoS One 8:e55458. doi: 10.1371/journal.pone.0055458

Saldaña, M. A., Etebari, K., Hart, C. E., Widen, S. G., Wood, T. G., Thangamani, S., et al. (2017). Zika virus alters the microRNA expression profile and elicits an RNAi response in Aedes aegypti mosquitoes. PLoS Negl. Trop. Dis. 11:e0005760. doi: 10.1371/journal.pntd.0005760 
Samuel, G. H., Adelman, Z. N., and Myles, K. M. (2018). Antiviral immunity and virus-mediated antagonism in disease vector mosquitoes. Trends Microbiol. 26, 447-461. doi: 10.1016/j.tim.2017.12.005

Samuel, G. H., Wiley, M. R., Badawi, A., Adelman, Z. N., and Myles, K. M. (2016). Yellow fever virus capsid protein is a potent suppressor of RNA silencing that binds double-stranded RNA. Proc. Natl. Acad. Sci. U.S.A. 113, 13863-13868. doi: 10.1073 /pnas.1600544113

Sánchez-Vargas, I., Scott, J. C., Poole-Smith, B. K., Franz, A. W. E., BarbosaSolomieu, V., Wilusz, J., et al. (2009). Dengue virus type 2 infections of Aedes aegypti are modulated by the mosquito's RNA interference pathway. PLoS Pathog. 5:e1000299. doi: 10.1371/journal.ppat.1000299

Sanders, H. R., Foy, B. D., Evans, A. M., Ross, L. S., Beaty, B. J., Olson, K. E., et al. (2005). Sindbis virus induces transport processes and alters expression of innate immunity pathway genes in the midgut of the disease vector, Aedes aegypti. Insect Biochem. Mol. Biol. 35, 1293-1307. doi: 10.1016/j.ibmb.2005.07.006

Sanjuan, R., Nebot, M. R., Chirico, N., Mansky, L. M., and Belshaw, R. (2010). Viral mutation rates. J. Virol. 84, 9733-9748. doi: 10.1128/JVI.00694-10

Santos, D., Vanden Broeck, J., and Wynant, N. (2014). Systemic RNA interference in locusts: reverse genetics and possibilities for locust pest control. Curr. Opin. Insect Sci. 6, 9-14. doi: 10.1016/j.cois.2014.09.013

Schmitt-Engel, C., Schultheis, D., Schwirz, J., Ströhlein, N., Troelenberg, N., Majumdar, U., et al. (2015). The iBeetle large-scale RNAi screen reveals gene functions for insect development and physiology. Nat. Commun. 6:7822. doi: 10.1038/ncomms8822

Schnettler, E., Donald, C. L., Human, S., Watson, M., Siu, R. W. C., McFarlane, M., et al. (2013a). Knockdown of piRNA pathway proteins results in enhanced Semliki Forest virus production in mosquito cells. J. Gen. Virol. 94, 1680-1689. doi: 10.1099/vir.0.053850-0

Schnettler, E., Ratinier, M., Watson, M., Shaw, A. E., McFarlane, M., Varela, M., et al. (2013b). RNA interference targets Arbovirus replication in Culicoides cells. J. Virol. 87, 2441-2454. doi: 10.1128/JVI.02848-12

Schnettler, E., Sreenu, V. B., Mottram, T., and McFarlane, M. (2016). Wolbachia restricts insect-specific flavivirus infection in Aedes aegypti cells. J. Gen. Virol. 97, 3024-3029. doi: 10.1099/jgv.0.000617

Schnettler, E., Sterken, M. G., Leung, J. Y., Metz, S. W., Geertsema, C., Goldbach, R. W., et al. (2012). Noncoding flavivirus RNA displays RNA interference suppressor activity in insect and mammalian cells. J. Virol. 86, 13486-13500. doi: 10.1128/JVI.01104-12

Schnettler, E., Tykalová, H., Watson, M., Sharma, M., Sterken, M. G., Obbard, D. J., et al. (2014). Induction and suppression of tick cell antiviral RNAi responses by tick-borne flaviviruses. Nucleic Acids Res. 42, 9436-9446. doi: 10.1093/nar/ gku657

Schultz, M. J., Frydman, H. M., and Connor, J. H. (2018). Dual Insect specific virus infection limits Arbovirus replication in Aedes mosquito cells. Virology 518, 406-413. doi: 10.1016/j.virol.2018.03.022

Schuster, S., Zirkel, F., Kurth, A., van Cleef, K. W. R., Drosten, C., van Rij, R. P., et al. (2014). A unique nodavirus with novel features: mosinovirus expresses two subgenomic RNAs, a capsid gene of unknown origin, and a suppressor of the antiviral RNA interference pathway. J. Virol. 88, 13447-13459. doi: 10.1128/ JVI.02144-14

Scott, J. C., Brackney, D. E., Campbell, C. L., Bondu-Hawkins, V., Hjelle, B., Ebel, G. D., et al. (2010). Comparison of dengue virus type 2-specific small RNAs from RNA interference-competent and -incompetent mosquito cells. PLoS Negl. Trop. Dis. 4:e848. doi: 10.1371/journal.pntd.0000848

Scott, J. G., Michel, K., Bartholomay, L. C., Siegfried, B. D., Hunter, W. B., Smagghe, G., et al. (2013). Towards the elements of successful insect RNAi. J. Insect Physiol. 59, 1212-1221. doi: 10.1016/j.jinsphys.2013.08.014

Senti, K. A., and Brennecke, J. (2010). The piRNA pathway: a fly's perspective on the guardian of the genome. Trends Genet. 26, 499-509. doi: 10.1016/j.tig.2010. 08.007

Siu, R. W. C., Fragkoudis, R., Simmonds, P., Donald, C. L., Chase-Topping, M. E., Barry, G., et al. (2011). Antiviral RNA interference responses induced by Semliki forest virus infection of mosquito cells: characterization, origin, and frequency-dependent functions of virus-derived small interfering RNAs. J. Virol. 85, 2907-2917. doi: 10.1128/JVI.02052-10

Skalsky, R. L., Olson, K. E., Blair, C. D., Garcia-Blanco, M. A., and Cullen, B. R. (2014). A "microRNA-like" small RNA expressed by Dengue virus? Proc. Natl. Acad. Sci. U.S.A. 111:E2359.
Slonchak, A., Hussain, M., Torres, S., Asgari, S., and Khromykh, A. A. (2014). Expression of mosquito MicroRNA Aae-miR-2940-5p is downregulated in response to west Nile virus infection to restrict viral replication. J. Virol. 88, 8457-8467. doi: 10.1128/JVI.00317-14

Smith, D. L., Battle, K. E., Hay, S. I., Barker, C. M., Scott, T. W., and McKenzie, F. E. (2012). Ross, macdonald, and a theory for the dynamics and control of mosquito-transmitted pathogens. PLoS Pathog. 8:e1002588. doi: 10.1371/ journal.ppat. 1002588

Soldan, S. S., Plassmeyer, M. L., Matukonis, M. K., and Gonzalez-Scarano, F. (2005). La Crosse virus nonstructural protein NSs counteracts the effects of short interfering RNA. J. Virol. 79, 234-244. doi: 10.1128/jvi.79.1.234244.2005

Souza-Neto, J. A., Sim, S., and Dimopoulos, G. (2009). An evolutionary conserved function of the JAK-STAT pathway in anti-dengue defense. Proc. Natl. Acad. Sci. U.S.A. 106, 17841-17846. doi: 10.1073/pnas.0905 006106

Stapleford, K. A., Rozen-Gagnon, K., Das, P. K., Saul, S., Poirier, E. Z., Blanc, H., et al. (2015). Viral polymerase-helicase complexes regulate replication fidelity to overcome intracellular nucleotide depletion. J. Virol. 89, 11233-11244. doi: 10.1128/JVI.01553-15

Swevers, L., Liu, J., and Smagghe, G. (2018). Defense mechanisms against viral infection in Drosophila: RNAi and non-RNAi. Viruses 10:E230. doi: 10.3390/ v10050230

Szemiel, A. M., Failloux, A. B., and Elliott, R. M. (2012). Role of Bunyamwera orthobunyavirus NSs protein in infection of mosquito cells. PLoS Negl. Trop. Dis. 6:e1823. doi: 10.1371/journal.pntd.0001823

Taning, C. N. T., Christiaens, O., Li, X., Swevers, L., Casteels, H., Maes, M., et al. (2018). Engineered flock house virus for targeted gene suppression through RNAi in fruit flies (Drosophila melanogaster) in vitro and in vivo. Front. Physiol. 9:805. doi: 10.3389/fphys.2018.00805

te Velthuis, A. J. (2014). Common and unique features of viral RNA-dependent polymerases. Cell. Mol. Life Sci. 71, 4403-4420. doi: 10.1007/s00018-0141695-z

Trobaugh, D. W., Gardner, C. L., Sun, C., Haddow, A. D., Wang, E., Chapnik, E., et al. (2014). RNA viruses can hijack vertebrate microRNAs to suppress innate immunity. Nature 506, 245-248. doi: 10.1038/nature 12869

Umbach, J. L., and Cullen, B. R. (2009). The role of RNAi and microRNAs in animal virus replication and antiviral immunity. Genes Dev. 23, 1151-1164. doi: 10.1101/gad.1793309

van Cleef, K. W. R., van Mierlo, J. T., Miesen, P., Overheul, G. J., Fros, J. J., Schuster, S., et al. (2014). Mosquito and Drosophila entomobirnaviruses suppress dsRNA- and siRNA-induced RNAi. Nucl. Acid. Res. 42, 8732-8744. doi: $10.1093 /$ nar/gku528

Varjak, M., Dietrich, I., Sreenu, V. B., Till, B. E., Merits, A., Kohl, A., et al. (2018a). Spindle-E acts antivirally against alphaviruses in mosquito cells. Viruses 10:E88. doi: 10.3390/v10020088

Varjak, M., Leggewie, M., and Schnettler, E. (2018b). The antiviral piRNA response in mosquitoes? J. Gen. Virol. 99, 1551-1562. doi: 10.1099/jgv.0. 001157

Varjak, M., Donald, C. L., Mottram, T. J., Sreenu, V. B., Meris, A., Maringer, K., et al. (2017a). Characterization of the Zika virus induced small RNA response in Aedes aegypti cells. PLoS Negl. Trop. Dis. 11:e0006010. doi: 10.1371/journal. pntd.0006010

Varjak, M., Maringer, K., Watson, M., Sreenu, V. B., Fredericks, A. C., Pondeville, E., et al. (2017b). Aedes aegypti Piwi4 is a noncanonical PIWI protein involved in antiviral responses. $m$ Sphere $2: \mathrm{e} 00144-17$. doi: $10.1128 / \mathrm{mSphere} .00$ 144-17

Vasilakis, N., Forrester, N. L., Palacios, G., Nasar, F., Savji, N., Rossi, S. L., et al. (2013). Negevirus: a proposed new taxon of insect-specific viruses with wide geographic distribution. J. Virol. 87, 2475-2488. doi: 10.1128/JVI.00 776-12

Vodovar, N., Bronkhorst, A. W., van Cleef, K. W. R., Miesen, P., Blanc, H., van Rij, R., et al. (2012). Arbovirus-derived piRNAs exhibit a ping-pong signature in mosquito cells. PLoS One 7:e30861. doi: 10.1371/journal.pone.0030861

Waldock, J., Olson, K. E., and Christophides, G. K. (2012). Anopheles gambiae antiviral immune response to systemic O'nyong-nyong infection. PLoS Negl. Trop. Dis. 6:e1565. doi: 10.1371/journal.pntd.0001565 
Wang, Y., Jin, B., Liu, P., Li, J., Chen, X., and Gu, J. (2018). piRNA profiling of dengue virus type 2-infected Asian tiger mosquito and midgut tissues. Viruses 10:E213. doi: 10.3390/v10040213

Weger-Lucarelli, J., Rückert, C., Grubaugh, N. D., Misencik, M. J., Armstrong, P. M., Stenglein, M. D., et al. (2018). Adventitious viruses persistently infect three commonly used mosquito cell lines. Virology 521, 175-180. doi: 10.1016/ j.virol.2018.06.007

Whitfield, Z. J., Dolan, P. T., Kunitomi, M., Tassetto, M., Seetin, M. G., Oh, S., et al. (2017). The diversity, structure, and function of heritable adaptive immunity sequences in the Aedes aegypti genome. Curr. Biol. 27, 3511-3519. doi: 10.1016/j.cub.2017.09.067

Whitten, M., and Dyson, P. (2017). Gene silencing in non-model insects: overcoming hurdles using symbiotic bacteria for trauma-free sustainable delivery of RNA interference: sustained RNA interference in insects mediated by symbiotic bacteria: applications as a genetic tool and as a biocide. Bioessays 39:1600247. doi: 10.1002/bies.201600247

Xi, Z., Ramirez, J. L., and Dimopoulos, G. (2008). The Aedes aegypti toll pathway controls dengue virus infection. PLoS Pathog. 4:e1000098. doi: 10.1371/journal. ppat. 1000098

Xing, S., Du, J., Gao, S., Tian, Z., Zheng, Y., Liu, G., et al. (2016). Analysis of the miRNA expression profile in an Aedes albopictus cell line in response to bluetongue virus infection. Infect. Genet. Evol. 39, 74-84. doi: 10.1016/j.meegid. 2016.01.012

Zhang, G., Asad, S., Khromykh, A. A., and Asgari, S. (2017). Cell fusing agent virus and dengue virus mutually interact in Aedes aegypti cell lines. Sci. Rep. 7:6935. doi: $10.1038 / \mathrm{s} 41598-017-07279-5$
Zhang, G., Hussain, M., O’Neill, S. L., and Asgari, S. (2014). Wolbachia uses a host microRNA to regulate transcripts of a methyltransferase, contributing to dengue virus inhibition in Aedes aegypti. Proc. Natl. Acad. Sci. U.S.A. 110, 10276-10281. doi: 10.1073/pnas.1303603110

Zhang, H., Li, H. C., and Miao, X. X. (2013). Feasibility, limitation and possible solutions of RNAi-based technology for insect pest control. Insect Sci. 20, 15-30. doi: 10.1111/j.1744-7917.2012.01513.x

Zhou, Y., Liu, Y., Yan, H., Li, Y., Zhang, H., Xu, J., et al. (2014). miR-281, an abundant midgut-specific miRNA of the vector mosquito Aedes albopictus enhances dengue virus replication. Parasit. Vectors 7:488. doi: 10.1186/s13071014-0488-4

Zirkel, F., Roth, H., Kurth, A., Drosten, C., Ziebuhr, J., and Junglen, S. (2013). Identification and characterization of genetically divergent members of the newly established family Mesoniviridae. J. Virol. 87, 6346-6358. doi: 10.1128/ JVI.00416-13

Conflict of Interest Statement: The authors declare that the research was conducted in the absence of any commercial or financial relationships that could be construed as a potential conflict of interest.

Copyright $\odot 2019$ Liu, Swevers, Kolliopoulou and Smagghe. This is an open-access article distributed under the terms of the Creative Commons Attribution License (CC BY). The use, distribution or reproduction in other forums is permitted, provided the original author(s) and the copyright owner(s) are credited and that the original publication in this journal is cited, in accordance with accepted academic practice. No use, distribution or reproduction is permitted which does not comply with these terms. 